\title{
SFMR Surface Wind Undersampling over the Tropical Cyclone Life Cycle
}

\author{
BRADLEY W. KLOTZ ${ }^{\mathrm{a}}$ \\ Cooperative Institute for Marine and Atmospheric Studies, University of Miami, and NOAA/AOML/Hurricane \\ Research Division, Miami, Florida \\ DAVID S. NOLAN \\ Rosenstiel School of Marine and Atmospheric Science, University of Miami, Miami, Florida
}

(Manuscript received 17 August 2018, in final form 16 October 2018)

\begin{abstract}
Surface wind speeds in tropical cyclones are important for defining current intensity and intensification. Traditionally, airborne observations provide the best information about the surface wind speeds, with the Stepped Frequency Microwave Radiometer (SFMR) providing a key role in obtaining such data. However, the flight patterns conducted by hurricane hunter aircraft are limited in their azimuthal coverage of the surface wind field, resulting in an undersampling of the wind field and consequent underestimation of the peak 10-m wind speed. A previous study provided quantitative estimates of the average underestimate for a very strong hurricane. However, no broader guidance on applying a correction based on undersampling has been presented in detail. To accomplish this task, a modified observing system simulation experiment with five hurricane simulations is used to perform a statistical evaluation of the peak wind speed underestimate over different stages of the tropical cyclone life cycle. Analysis of numerous simulated flights highlights prominent relationships between wind speed undersampling and storm size, where size is defined by the radius of maximum wind speed (RMW). For example, an intense hurricane with small RMW needs negligible correction, while a large-RMW tropical storm requires a 16\%-19\% change. A lookup table of undersampling correction factors as a function of peak SFMR wind speed and RMW is provided to assist the tropical cyclone operations community. Implications for hurricane best track intensity estimates are also discussed using real data from past Atlantic hurricane seasons.
\end{abstract}

\section{Introduction}

Invaluable strides toward improving tropical cyclone (TC) track and intensity forecasts have been made over the past few decades. While track forecasts have steadily improved (Gall et al. 2013), intensity forecasts are improving more slowly due to deficiencies in the understanding of physical processes in TCs and the ability to model those processes in numerical simulations (DeMaria et al. 2014). As part of this desire to understand the deficiencies in TC forecasting, data assimilation systems (i.e., Aksoy et al. 2012, 2013) are designed to incorporate various observations from satellite-, aircraft-, ocean-, and land-based

\footnotetext{
${ }^{\text {a }}$ Current affiliation: Applied Research Laboratories, The University of Texas at Austin, Austin, Texas.
}

Corresponding author: Dr. Bradley Klotz, brad.klotz@arlut. utexas.edu instruments, often evaluating the effects on the predicted TC track, intensity, three-dimensional structure, and surrounding environment by including or omitting certain observations (Aberson et al. 2015; Christophersen et al. 2017). Assimilation and prediction systems rely heavily on airborne and satellite data, and sophisticated satellite instruments indicate specific details about precipitation, wind, or thermodynamic structure in all TC basins. Determining direct TC intensity estimates, however, remains a shortcoming of spaceborne platforms that struggle to identify small-scale features related to storm intensity. Therefore, in situ and remote sensing observations from hurricanepenetrating aircraft remain the most accurate and preferable source of intensity information for forecasters.

Operational forecast centers report TC intensity based on estimates of the maximum 1- or 10-min average wind speed at 10-m height above the surface (OFCM 2012) in the form of a "best track" estimate (Jarvinen et al. 1984). The best track intensities are derived from multiple data 
sources over a 6-h window at synoptic times (Landsea and Franklin 2013). There is an inherent inability to observe this metric or resolve it in numerical simulations (Vukicevic et al. 2014). There are also subjective influences on the estimate that affect comparisons of observational or model data to it. The uncertainty of this intensity measure is $5 \mathrm{~m} \mathrm{~s}^{-1}$ (Landsea and Franklin 2013), which is slightly lower for weak tropical storms and higher for strong hurricanes. Despite these caveats, the best track intensity is a reasonable baseline value to assess the current strength of a TC. In the absence of aircraft data, TC intensity is estimated from the satellite technique developed by Dvorak $(1975,1984)$. While the Dvorak method is extremely useful, it is known to have larger errors for storms that reach category 4 or higher intensity on the Saffir-Simpson hurricane wind scale (Knaff et al. 2010; Cangialosi et al. 2015). Hurricane hunter aircraft, however, are fitted with the Stepped Frequency Microwave Radiometer (SFMR; Uhlhorn and Black 2003; Uhlhorn et al. 2007; Klotz and Uhlhorn 2014) that provides surface wind speed and rain rate directly below the aircraft flight track. Throughout this study, reference to the SFMR surface wind speed is interpreted as a representative $10-\mathrm{m}$ wind speed. Despite the limited spatial coverage, the SFMR is capable of providing reliable estimates of surface wind speeds $>10 \mathrm{~m} \mathrm{~s}^{-1}$. While the improvements to the SFMR algorithm described by Klotz and Uhlhorn (2014) reduced high biases at the low wind speeds in precipitation (tropical storm winds), they were not completely removed. This remaining high bias must be taken into consideration when using SFMR data.

In a given storm, coverage of the full 10-m wind field by the SFMR is sparse due to the design of the flight patterns and small footprint. A previous study from Landsea et al. (2004) also describes undersampling by dropsondes (Franklin et al. 2003) and discusses use of a flight-level wind speed reduction to obtain surface wind speed estimates, where the surface wind speed is $80 \%-90 \%$ of the flight-level wind speed (i.e., Powell and Black 1990). Powell et al. (2009) wanted to revisit the premise of the reduction factor because SFMR winds had been collected over multiple hurricane seasons and could be used to develop more accurate comparisons. They performed an analysis of the slope-dependent flight-level reduction factor and developed several ways to estimate the maximum surface wind speed through linear regression techniques. They concluded that the average reduction factor was close to $83 \%$ with azimuthal variability and also determined that the " $90 \%$ rule" was biased high. While they used a reliable set of SFMR winds, it remains that the reported peak wind speed from SFMR underestimates the true maximum surface wind speed. With reliance on aircraft data that undersample a $10-\mathrm{m}$ wind field, it is expected that the best track intensity will also underestimate the 1-min peak wind within the wind field. To clarify, any reference to undersampling refers to the ability (or lack thereof) of an observing system to adequately sample a TC wind field. An underestimate is a consequence of the undersampling because the likelihood of observing the absolute peak wind speed is statistically close to zero. To quantify the underestimate, the best practice is to compare observations to the spatially averaged wind speed within a given time period rather than an estimate of the absolute peak. Because Uhlhorn and Nolan (2012) utilized this technique, it is known that the SFMR underestimates the 1-min and 6-h (i.e., best track) peak wind speed. However, application of any adjustment of surface wind speeds in the best track due to undersampling of the wind field is currently subjective and lacks formal guidance. Use of the terminology for peak wind speeds in the remainder of this article will refer to the 1-min average wind speed unless otherwise specified.

From the variety of sampling patterns or inconsistencies in data, it is somewhat difficult to provide guidance on a subject that has not been objectively detailed. One way to evaluate the strategies employed in current operational aircraft missions is to perform a modified version of an observing system simulation experiment (OSSE; Arnold and Dey 1986; Atlas 1997). In this type of experiment, a current or potential observing system can be simulated in a numerical model for the purposes of validating sampling strategies, model performance, and data assimilation techniques. The latter two of these examples are beyond the scope of this work, and our main focus is to evaluate the existing hurricane hunter sampling strategies.

Uhlhorn and Nolan (2012) employed an OSSE-like experiment to quantify the maximum wind speed underestimate from hypothetical SFMR observations. Using a simulation of Hurricane Isabel from the 2003 Atlantic season when it was intense and symmetric (Nolan et al. 2009a,b), they discovered that under the constraints of the typical operational flight patterns, one should expect, on average, a $7 \%-10 \%$ peak 1 -min wind speed underestimate. For their most favorable sampling patterns, the wind speed underestimate decreased to $\sim 3.5 \%-4 \%$, but there was only a one-in-eight chance for this to occur. Distributions of the peak winds within the eyewall of Hurricane Isabel indicate that $95 \%$ of these wind speeds are $<90 \%$ of the peak 1-min wind.

A follow-up study from Nolan et al. (2014) employed a similar type of experiment by first defining a "perfect" observing system as a dense grid of surface anemometers. For the most common case of a single surface measurement in a "perfect" location (i.e., a direct hit by the rightfront eyewall), the maximum observed wind speed by such an instrument underestimates the true maximum in the same time interval typically by $5 \%-10 \%$, but also by as 
much as $18 \%$ during different stages of TC development. Figure 8 from their study shows that by adding ideally placed anemometers in the direct path of the peak winds, one or two anemometers observe peak wind speeds close to the mean or best track winds. However, this figure also indicates that surface anemometers will underestimate the peak wind speeds by at least $5 \mathrm{kt}\left(1 \mathrm{kt} \approx 0.5144 \mathrm{~m} \mathrm{~s}^{-1}\right)$ due to the model's inability to resolve small-scale turbulent features as they found by comparison to real observations from tower data.

While Nolan et al. (2014) present some compelling results, duplicating their anemometer network in reality is not feasible, which increases the importance of quantifying the underestimates expected from aircraft observing systems. The concepts of Uhlhorn and Nolan (2012) for a specific storm structure thus lead naturally to the question of how the SFMR underestimate varies at different stages of TC development, from changes in vortex structure or intensity, or from changes and improvements to the numerical simulations used in a modified OSSE. In terms of storm structure, it is possible to have variations in storm size or azimuthal and radial symmetry across TCs of similar intensity. These differences affect the determination of an azimuthally averaged radius of maximum wind speed (RMW) and also have an impact on the likelihood of sampling the peak surface winds. In addition, improvements to physics and boundary layer parameterizations along with higher-resolution grids allow models to better replicate surface wind speeds and their variability through the two-dimensional horizontal wind field and provide a better medium to perform the modified OSSE experiments. Therefore, the purpose of this study is twofold: 1) evaluate and quantify the underestimate of the peak surface wind speed from SFMR-like observations over the TC life cycle using a trusted, high-resolution model framework; and 2) provide a more objective guide for applying a correction to the peak underestimated wind speed from SFMR. The sections of this article are as follows. The second section briefly discusses the model simulation data used for what is referred to throughout as the SFMR OSSE, and the third section describes the methodology and various experiments performed. The fourth section describes the experiment results, while the fifth section discusses application techniques with real observations. The final section provides some conclusions of the study with mention of ongoing or future related work.

\section{Simulation and data description}

\section{a. Model selection and details}

The goals of the current study are to document and quantify the variation in the surface wind undersampling over the TC life cycle, from initial development, intensification, maturation, and weakening phases. Each of this study's predecessors (Uhlhorn and Nolan 2012; Nolan et al. 2014) performed observation experiments using the output from a single numerical simulation of a hurricane. To expand the range of possible hurricane "states" as represented by their varying measures of size, intensity, and asymmetry, this study uses model outputs from five different simulations, most of which capture several stages of the hurricane life cycle. These are the hurricane nature run 1 (HNR1; Nolan et al. 2013), hurricane nature run 2 (HNR2; Nolan and Mattocks 2014), a simulation of Hurricane Bill (2009), and two idealized simulations of hurricanes with mean flow and wind shear. The two idealized simulations will be referred to as Ideal 3 and Ideal 5 for reasons described in appendix A. These simulations were chosen or constructed to be as similar as possible in terms of model, resolution, and physical parameterizations. Specifically, all five use the same grid spacing, vertical levels, and parameterizations of physical processes as HNR1. Further discussion of the rationale for using these simulations and additional details are provided in appendix A. Figure 1 provides the tracks for the respective model simulations, with the bold portion of the track coinciding with the selection used for the SFMR OSSE, which will be discussed in a subsequent section. Because of the substantial interaction with land in HNR2, only the period when it is between Cuba and south Florida is used.

\section{b. Storm characteristics}

To evaluate the undersampling of SFMR-like observations, it is important to consider multiple characteristics of the simulated storms. These parameters include peak wind speed, RMW, and asymmetric wind structure. Regarding the peak wind speed, it is not useful to rely on the instantaneous model output for the peak value because it will overestimate values typically used by operational centers to classify TC intensity (1- or 10-min averages). In an effort to convert the instantaneous wind speeds to these time-averaged values, Uhlhorn and Nolan (2012) loosely use the concept of a gust factor $\left(G_{i, T}\right)$, defined by the peak instantaneous wind speed in a given time window divided by the wind speed averaged over the 1- or 10-min period surrounding the maximum value. Traditional gust factors as described by Vickery and Skerlj (2005) are not generally reproducible in a model simulation due to resolution constraints. Uhlhorn and Nolan (2012) found their gust factors to be wind speed dependent and, for the 1-min conversion, $\sim 1 \%-2 \%$ less than the instantaneous value at wind speeds $>60 \mathrm{~m} \mathrm{~s}^{-1}$. The 10 -min conversion 


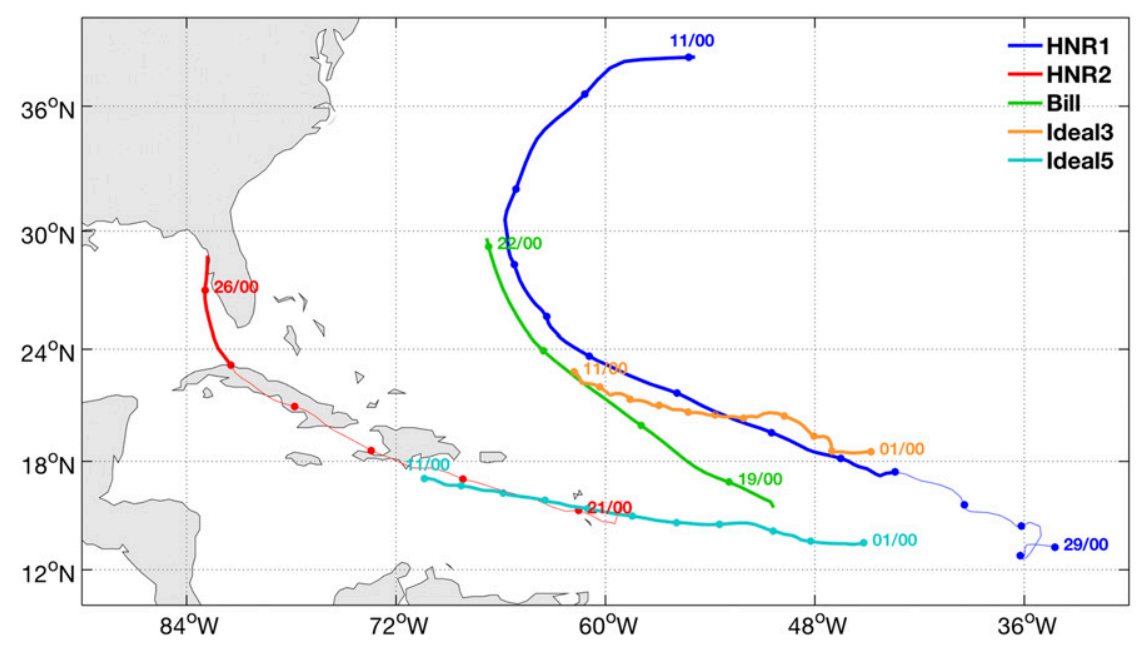

FIG. 1. Storm tracks for the five TC simulations are shown. The markers indicate the position at 0000 UTC on a particular date during the simulation. Thicker lines on the tracks highlight the portion of the simulation used in the current study. The tracks of the two idealized storms are included for comparison, with their initial latitudes on this map equal to the latitudes with Coriolis parameters equal to their $f$-plane values. The initial longitudes are arbitrary.

experiences a $2 \%$ change at $50 \mathrm{~m} \mathrm{~s}^{-1}$ and $5 \%-12 \%$ above $60 \mathrm{~m} \mathrm{~s}^{-1}$. For this current study, the same conversion factors are used to provide time averaging for the model $10-\mathrm{m}$ wind speed. The equations are as follows from Uhlhorn and Nolan (2012):

$$
G_{i, 1}=1+2.5 \times 10^{-10}\left(U_{i}-23\right)^{4.7}
$$

and

$$
G_{i, 10}=1+2.1 \times 10^{-11} U_{i}^{5.3},
$$

where $G_{i, 1}$ and $G_{i, 10}$ are the 1- and 10-min conversion factors, respectively, and $U_{i}$ is the instantaneous ( $i$ ) peak $10-\mathrm{m}$ wind speed (i.e., direct model wind speed output) at each grid point. To obtain the averaged wind speed, divide $U_{i}$ by $G_{i, T}$.

Operational assessments of intensity generally occur at synoptic times (i.e., 0000, 0600, 1200, and 1800 UTC), and data are considered from a variety of observational platforms. These wind speeds, which are evaluated over a 6-h window surrounding the synoptic time (Landsea and Franklin 2013), are initially reported in a "working best track" and are later applied to the official best track database after verification in a postseason analysis (Jarvinen et al. 1984; Landsea et al. 2004; Landsea and Franklin 2013). To evaluate the undersampling relative to the best track wind speed, a running average of the peak 1-min winds over a 6-h period is calculated similarly to Uhlhorn and
Nolan (2012). Figure 2 provides the time series of the peak 1-min and 6-h running mean wind speeds $\left(V_{\max }\right)$ for each model simulation, with the bold lines corresponding to the portion used for the SFMR OSSE.

Storm size and structure are also vital characteristics to consider for impacts on SFMR peak wind speed underestimates. Figure 3 shows examples of instantaneous $10-\mathrm{m}$ wind fields from the various simulations and highlights the structural range obtained throughout the model data sample. The top three panels are snapshots from HNR1 showing a disorganized tropical storm, a strong hurricane with a large wind field, and a slowly weakening hurricane with an expanding wind field. The lower-left panel (from HNR2) displays the effects from land interaction, while the lower-middle and lower-right panels are from Bill and Ideal5, respectively, and indicate intense hurricanes with small RMW.

These wind fields in Fig. 3 show a variety of interesting wind structures with spatial scales ranging from 4 to $20 \mathrm{~km}$. Because there is currently no way to instantaneously observe the surface wind field of a hurricane to the required spatial resolution, it is difficult to know whether these structures are realistic. The localized wind maxima in the eyewalls compare reasonably well to the larger, coherent structures observed by landbased and airborne radar and in situ measurements (Aberson et al. 2006; Marks et al. 2008; Hendricks et al. 2012). Outside of the eyewall, some of the features are caused by convective downdrafts, whereas others appear to be wind streaks associated with boundary 



FIG. 2. The 1-min mean and 6-h mean intensity $\left(V_{\max }\right)$ time series are shown for each of the five model simulations. The thick line in each panel corresponds to the track section highlighted in Fig. 1. The 0000 UTC time on a particular date is noted along the top axis, while the forecast hour is noted in the bottom axis.

layer rolls that are likely underresolved. In some cases (e.g., Figs. 3b,c), wind streaks are at an outward angle, compared to the direction of the low-level flow. Direct observations of wind streaks by portable Doppler radar find them generally to be parallel to the local wind or oriented at an inward angle (Wurman and Winslow 1998; Lorsolo et al. 2008; Kosiba and Wurman 2014). However, these observations were all taken over or very close to land, which can significantly change the low-level wind profile and affects the preferred orientations of the bands (Nolan 2005). Large-eddy simulations of a hurricane by Green and Zhang (2015) also show outwardoriented wind streaks even as the resolution is decreased to $111 \mathrm{~m}$. Fortunately, the wind streaks seen in the simulations only appear when the simulated storms are intense, and during this time, the peak winds always occur in the eyewall. Therefore, the wind streaks outside the inner core do not contribute to the undersampling effects that will be shown later. This point, as well as a comparison of the wind fields using different horizontal and vertical diffusion schemes, is further discussed in appendix B.

Highlighting the differences between a minimal and an intense hurricane, Fig. 4 shows simulated SFMR profiles through the wind fields from Figs. 3b, 3d, and 3f. The simulated flight paths for each of these profiles are southwest to northeast, west to east, and north to south, respectively. Paths were chosen based on the location of the maximum model surface wind speed during the simulated flight. The profile for HNR2 indicates the expected large storm with gradually decreasing wind speeds outward from the center. The comparison of HNR1 to Ideal5 shows that hurricanes that are of similar strength can have vastly different radial profiles of wind speed and overall wind structure. The wind speed drops sharply beyond the maximum in Ideal5, while HNR1 has a more gradual decrease. Also evident is the symmetry difference between HNR1 and Ideal5, where for the former, the peak wind speeds on the two sides of the eye vary by $15 \mathrm{~m} \mathrm{~s}^{-1}$, while the latter vary by only $5 \mathrm{~m} \mathrm{~s}^{-1}$.

Size and symmetry are quantifiable terms and are conveyed by the RMW and an asymmetry factor. The size term here is not necessarily representative of the full vortex size, but a larger RMW tends to correspond to a larger vortex. The extent of the vortex is often defined by the radius of minimal tropical storm force winds (i.e., $>17 \mathrm{~m} \mathrm{~s}^{-1}$; Chavas and Emanuel 2010), but this definition of size is less relevant to the present study. To determine the RMW from the two-dimensional surface winds, azimuthally averaged wind speeds are produced, and the location of the maximum wind indicates the RMW. The asymmetry of the wind field is determined by first taking the azimuthal-mean wind speed within $\pm 12 \mathrm{~km}$ of the RMW and then calculating the average Fourier decomposed wavenumber 1 and 2 magnitude 

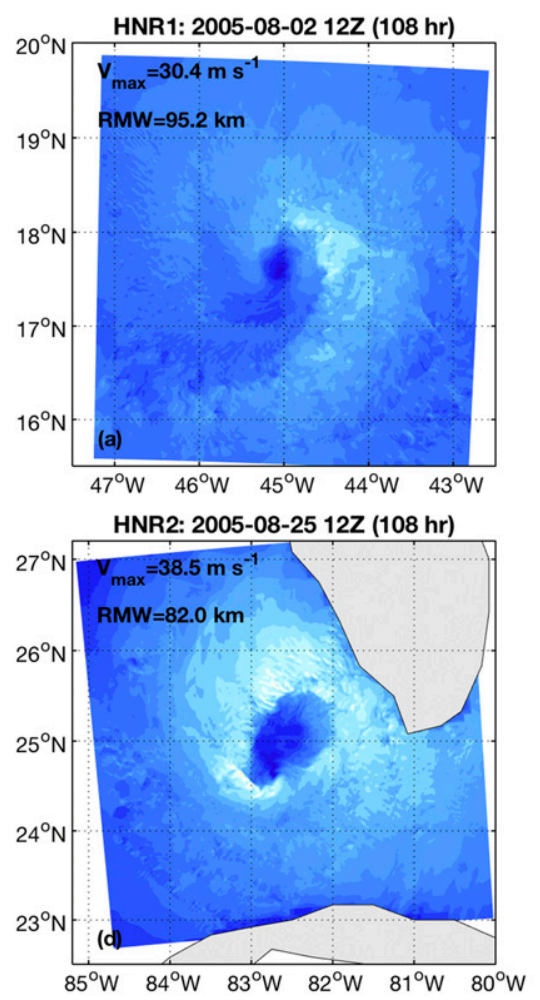


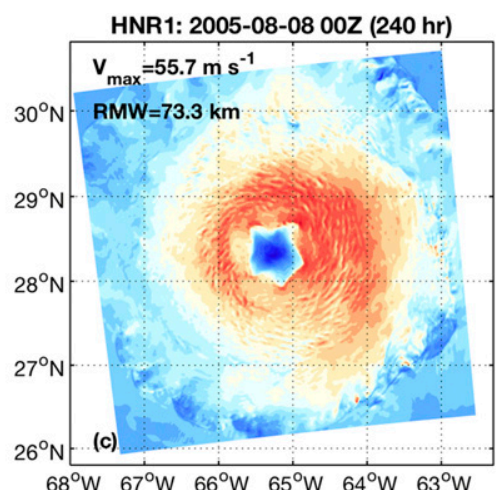

Ideal5: 2015-09-05 00Z (96 hr)

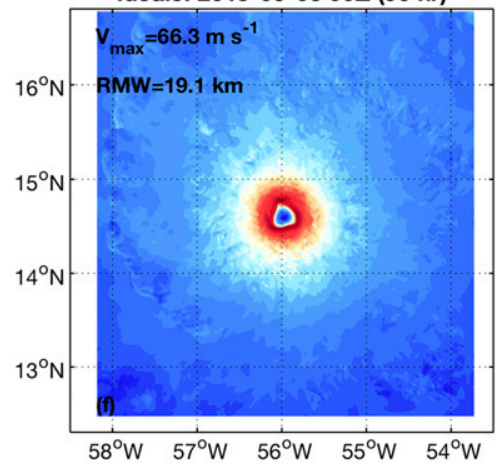

10

30

40

50

60

FIG. 3. Examples of the 10-m wind field $\left(\mathrm{m} \mathrm{s}^{-1}\right)$ are shown for various stages of the TC life cycle. (a)-(c) The tropical storm, intense hurricane, and slowly weakening hurricane with expanding wind field are shown from HNR1. (d) An example of land interaction from HNR2, and strong hurricanes with small RMW from (e) Bill and (f) Ideal5. The peak wind speed $V_{\max }$ and RMW are indicated in each panel.

within the same annulus. The asymmetry factor is then calculated using the following equation:

$$
A=\frac{\sqrt{f_{1}^{2}+f_{2}^{2}}}{V_{m}}
$$

where $f_{1}$ and $f_{2}$ are the amplitudes of the first two wavenumbers of the Fourier decomposition, and $V_{m}$ is the mean wind speed within the annulus around the RMW. Figure 5 shows the RMW and asymmetry factor $A$ for the five simulations in a similar manner to Fig. 2. As expected, decreasing RMW is generally correlated with decreasing asymmetry (i.e., lower $A$ ), but the difference between HNR1 and Ideal5 depicted in Fig. 4 is muted here due to the azimuthal averaging.

It is apparent that there is a connection between RMW and intensity, and theoretical studies from Shapiro and Willoughby (1982) and Willoughby et al. (1982) suggest that storm intensification and RMW contraction occur at the same time. Kimball and Mulekar (2004) use the existing extended best track dataset to examine storm size changes associated with intensification, and their results tend to support the ideas of the two theoretical studies above. However, Vigh (2010) uses a combination of best track and aircraft flight-level RMW to suggest that substantial contraction of the RMW occurs prior to eye formation, and storms that continue to intensify tend to see, at most, a slow contraction of the RMW. Stern et al. (2015) evaluate the contraction principle with idealized TC simulations in WRF and develop a method for determining the rate of RMW change. Their model results indicate that shrinking of the RMW slows after rapid intensification until eventually remaining steady for the remainder of the simulation even as intensity continues to increase. Using an observational flightlevel dataset (Vigh et al. 2012), Stern et al. (2015) show in their Fig. 12 that RMW contraction is greatest at the initial stages of intensification, while significantly slowing at wind speeds exceeding $65 \mathrm{kt}$. In contrast, the RMW expands during secondary eyewall formation and eyewall replacement cycles (Willoughby et al. 1982; Black and Willoughby 1992), acting to enlarge the vortex and slow intensification rates. 


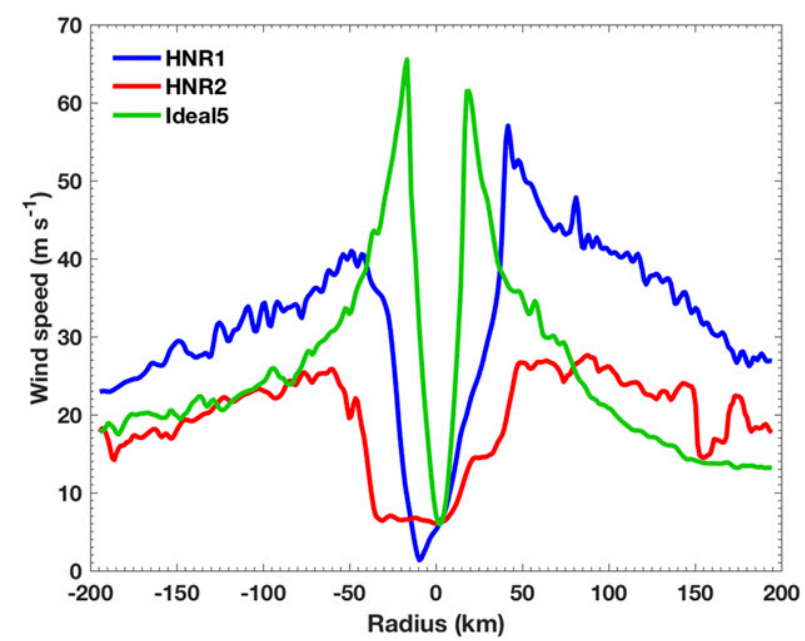

FIG. 4. Radial profiles of simulated surface wind speeds for the model examples in Figs. 3b, 3d, and 3f. The respective profiles are produced from transects from southwest to northeast, west to east, and north to south.

From the five simulations in the current study, the 10-m RMW and peak 1-min mean model wind speed are compared. For the entire intensity spectrum, RMW and intensity have a moderate negative correlation with a correlation coefficient of -0.7 . Once wind speed exceeds $40 \mathrm{~m} \mathrm{~s}^{-1}$, this correlation magnitude increases to -0.4 . The better correlation associated with weaker wind speed is a consequence of disorganized systems consolidating as they intensify, which is most often associated with a decreasing RMW in the earlier stages. After consolidation, RMW has less of a connection with the overall intensity, in agreement with Stern et al. (2015) and Vigh (2010).

\section{c. Real SFMR data applications}

The simulated storms provide the best opportunity to determine a peak surface wind speed underestimate from the SFMR. Given the fact that SFMR winds are simulated in this study, it is important to remind the reader of the reliability of these winds. Uhlhorn and Nolan (2012) took a set of real SFMR observations and computed histograms of the peak wind speed anomaly that represented the difference between the sum of the Fourier decomposed wavenumber 0 and 1 value from the observed peak wind along a flight radial. The same procedure was completed in their simulated flights. They discovered the observed and simulated winds were almost identical to each other for single figure-four flight patterns, which are discussed in the next section. For rotated figure-four patterns that doubled the storm sampling, the simulated peak winds were distributed over a narrower band. Because of this difference, they developed a "calibration" of the simulated winds using histogram matching and showed that despite the difference with the rotated figure-four pattern, the
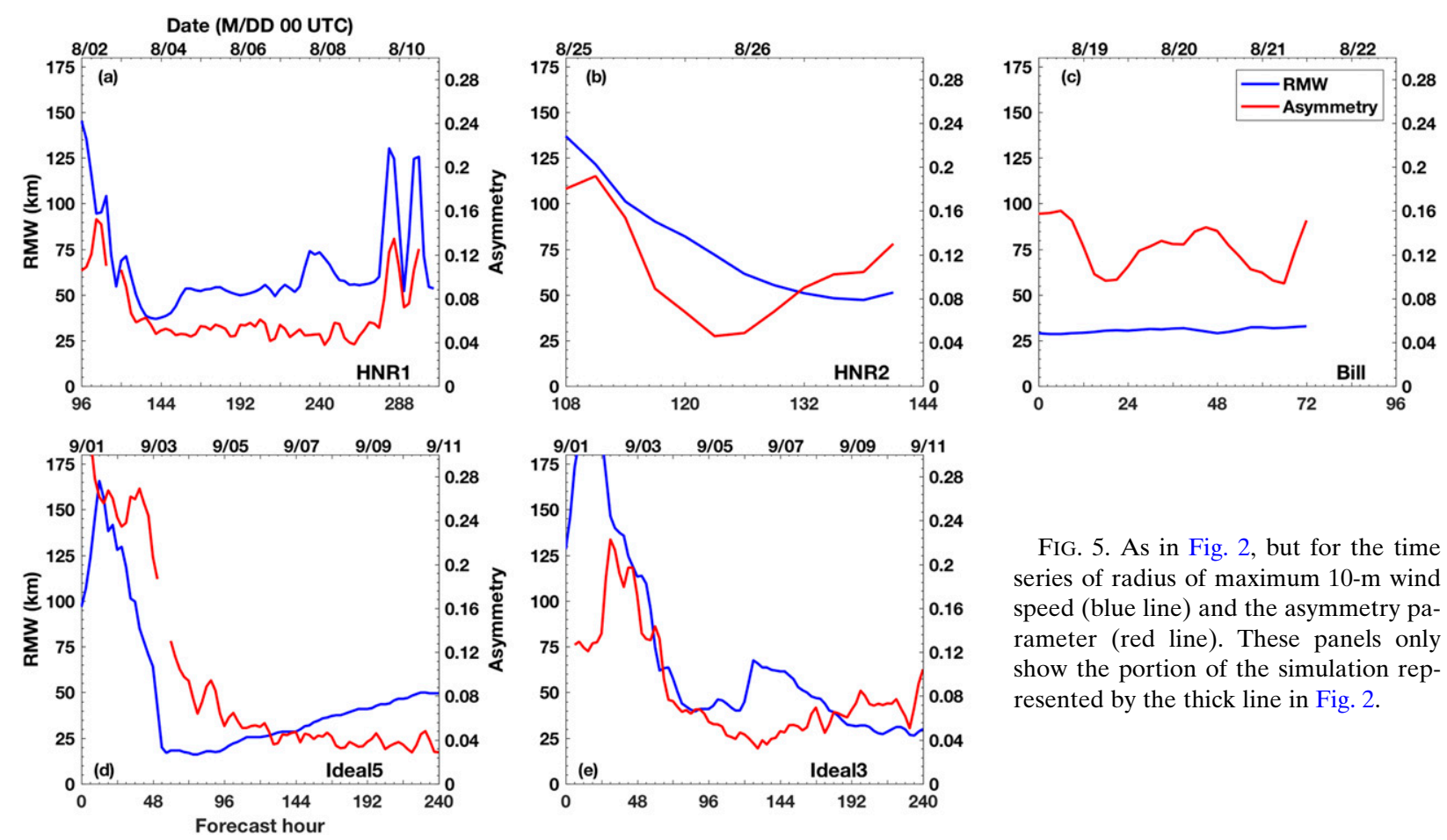

FIG. 5. As in Fig. 2, but for the time series of radius of maximum $10 \mathrm{-m}$ wind speed (blue line) and the asymmetry parameter (red line). These panels only show the portion of the simulation represented by the thick line in Fig. 2. 
simulated winds represented SFMR observations quite well.

Because the simulated winds are representative of real observations, the simulation results can be used in real cases to investigate the impacts of applying some amount of correction due to undersampling of the wind field. A random sample of cases from 1998 to 2016 that encapsulate the flight patterns recreated in the simulated flights is determined. Of the hundreds of missions operated by NOAA's Aircraft Operations Center in partnership with the Hurricane Research Division during their annual field program (Rogers et al. 2013, and references therein), 141 cases were selected for the validation dataset. The sample is separated by best track intensity as evenly as possible, with 55 tropical storms, 51 category 1 and 2 hurricanes, and 35 category 3 or higher hurricanes. For each case, the flight pattern, best track maximum wind speed, peak SFMR 1-min wind speed, and the RMW are recorded. The importance of these values will be discussed in a subsequent section.

\section{Methodology and experiment design}

\section{a. Simulating SFMR observations}

The methodology for simulating SFMR wind speeds from hurricane-penetrating aircraft is documented in detail by Uhlhorn and Nolan (2012), but a summary is given here. To adequately quantify the undersampling, flight patterns flown in operational missions are replicated in the SFMR OSSE. Traditional patterns include a single figure-four (alpha) pattern, a single butterfly pattern, and rotated or repeated figure-four patterns. Rotated figure-four patterns are operated in a manner such that eight equally spaced (azimuthally) radial profiles of the vortex are sampled, while the repeated figure-four samples the same four radials twice. Figure 6 provides examples of these flight patterns, where the underlying surface wind field (added here for illustration) is from HNR1 at 0000 UTC 4 August 2005. Each panel indicates that the successive patterns increase the coverage of the storm, where a single figure-four has minimal coverage and a rotated figure-four has maximum coverage allowed by operational constraints. The time series of simulated SFMR wind speed along the flight track is provided in the lower portion of each panel.

The radial distance of each flight transect is $196 \mathrm{~km}$ $(\sim 106$ nautical miles; $1 \mathrm{n} \mathrm{mi}=1.852 \mathrm{~km})$ from the center. The aircraft usually flies at $3-\mathrm{km}$ altitude at a speed of $230 \mathrm{kt}$, and the typical flight durations for the single figure-four, single butterfly, repeated figure-four, and rotated figure-four patterns are 2.5, 3.4, 5.6, and 5.3 h, respectively. The repeated figure-four has a longer duration than the rotated version because of the downwind rotation of $90^{\circ}$ relative to the end of the initial figure-four. The rotated figure-four requires only a $45^{\circ}$ rotation at this same point in the flight. A separate pattern not included in Fig. 6 is introduced here, referred to as a resampled figure-four. The resampled figure-four is similar to a single figure-four but adds two additional peak wind observations along the azimuth closest to the right-front quadrant in a storm motion-relative sense. Figure 7 provides a schematic of the resampled figure-four pattern and highlights the location resampled by the aircraft. The additional sampling extends $\sim 100 \mathrm{~km}$ from the center (i.e., half of the full radial transect) in an effort to capture any changes near the peak wind speed that may occur over a short time period.

The operational SFMR does not observe a single point beneath the aircraft flight track, but rather receives a signal over a $\sim 1.3-1.5-\mathrm{km}$ diameter footprint at nadir. Six C-band brightness temperatures are reported at a rate of $1 \mathrm{~Hz}$, and it typically takes $10 \mathrm{~s}$ to obtain a set of new brightness temperatures (Uhlhorn et al. 2007). Based on the aircraft speed, there are overlaps between successive observations; therefore, a 10-s average is usually calculated to obtain a 1-min wind speed (Powell et al.1991). The wind speed and column-averaged rain rate are then calculated from an inversion scheme within the SFMR geophysical model function. To replicate SFMR wind speeds for the SFMR OSSE, it is not necessary to apply the geophysical model function, but only the footprint size and time stepping techniques. Uhlhorn and Nolan (2012) describe using the half-power beamwidth and the $1 \%$ of peak normalized power as metrics for determining the footprint size, where any grid points that occur outside the $1 \%$ power threshold are excluded. As noted by Uhlhorn and Nolan (2012), the $1 \%$ power footprint size over $1 \mathrm{~s}$ of the flight would encapsulate three to five grid points, which are weighted based on distance to the aircraft. To obtain SFMR-like observations every $10 \mathrm{~s}$ for the 1 -min mean wind estimate, the phase-preserving filter that incorporates the temporal and spatial weighting scheme includes between seven and nine model grid points, with points closest to the aircraft getting the highest consideration in the wind calculation. For a single figure-four pattern, 888 simulated SFMR wind speed observations are generated through the spatial and temporal weighting of the winds along the flight track over 30 model time steps (25 for HNR1).

\section{b. Experiments and evaluation techniques}

To test the SFMR underestimate of TC peak surface wind speeds, flight patterns are conducted at eight initial 

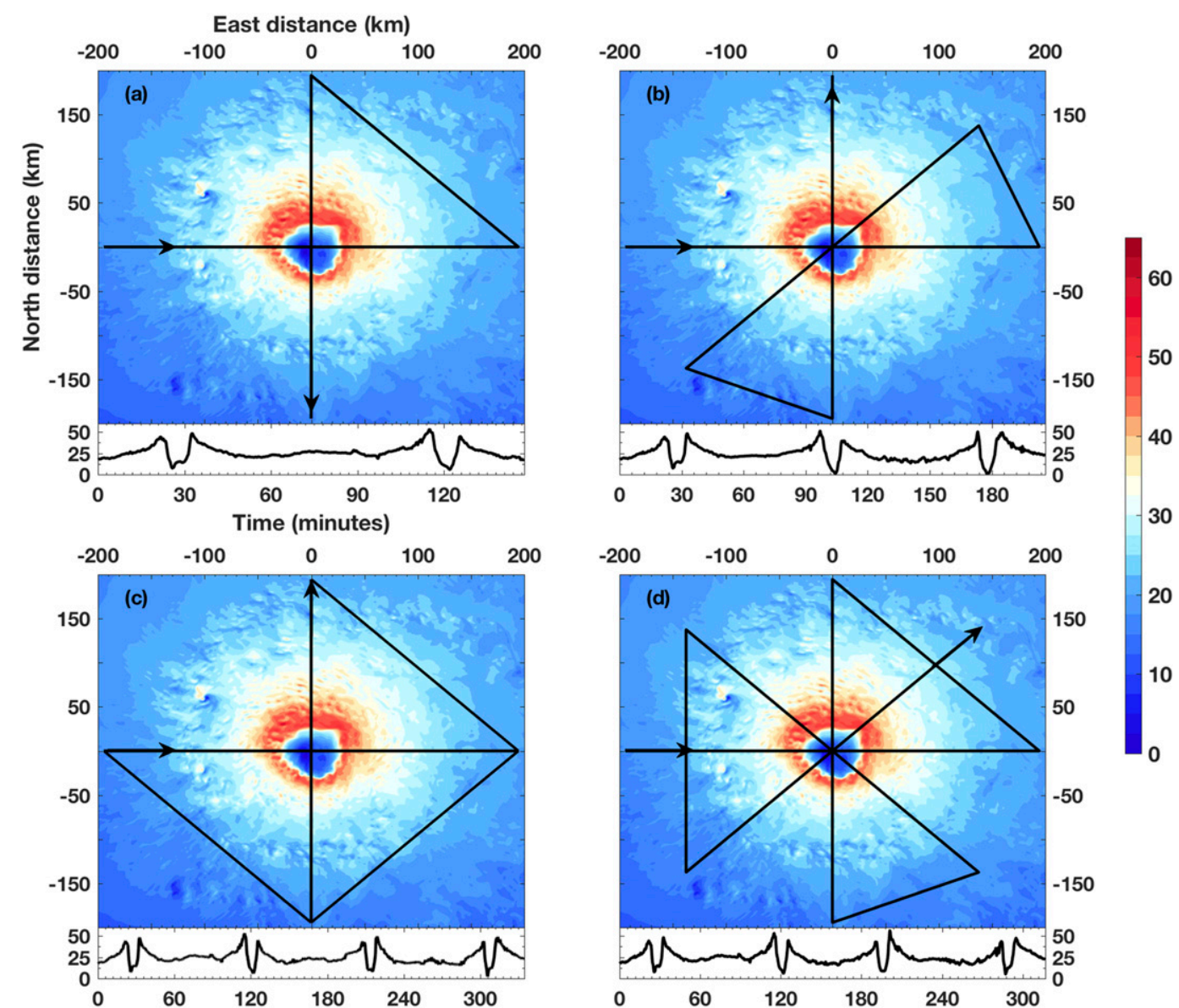

FIG. 6. Sample flight patterns used in this study are shown for the (a) single figure-four, (b) single butterfly, (c) repeated figure-four, and (d) rotated figure-four. The two arrows indicate the initial inbound and final outbound locations of each pattern. The underlying contour plots are taken from a single output time of HNR1 (0000 UTC 4 Aug 2005) to indicate the extent of the full wind field sampled by each pattern. The lower inset of each panel shows the time series of $10-\mathrm{m}$ wind speed $\left(\mathrm{m} \mathrm{s}^{-1}\right)$ that would be sampled directly beneath the aircraft. This time series is not sampled over the single wind field, but rather the temporal and spatial model output, as discussed in the text.

azimuths starting at $0^{\circ}$ and rotated every $45^{\circ}$ around the circle for each subsequent flight. Three-hour initial flight time separation is used to increase data in the sample. At each initial time, there are eight simulated flights for each pattern described previously, such that each flight starts at one of the eight azimuths described above. Over each 24-h period, 64 simulated flights are produced with flight times corresponding to the respective pattern chosen and within the respective model storm. Table 1 provides the total number of flights conducted and total flight time within each model storm simulation. From each flight, the peak SFMR-like wind speed and the peak instantaneous, 1-min, 10-min, and 6-h mean model wind speeds are obtained. The eight maximum SFMR wind speeds per flight time period are averaged to obtain a mean value that is then compared to the respective model maximum to determine the underestimate. The minimum underestimate and the
95\% confidence intervals are also obtained from the simulated SFMR peak wind speeds.

\section{Experiment results}

\section{a. Single figure-four and single butterfly}

The basic patterns flown include the single figure-four and single butterfly. The underestimate is first evaluated by comparing the mean SFMR peak winds to the 1-min mean model maximum within the flight time window. Additional comparisons between the SFMR and 10-min model peak winds [using the conversion factor in Eq. (2)] and the 1-min mean SFMR and 6-h best track peak winds are computed. The mean underestimate values associated with these comparisons are provided in Table 2 in wind speed and percentage units for the five 


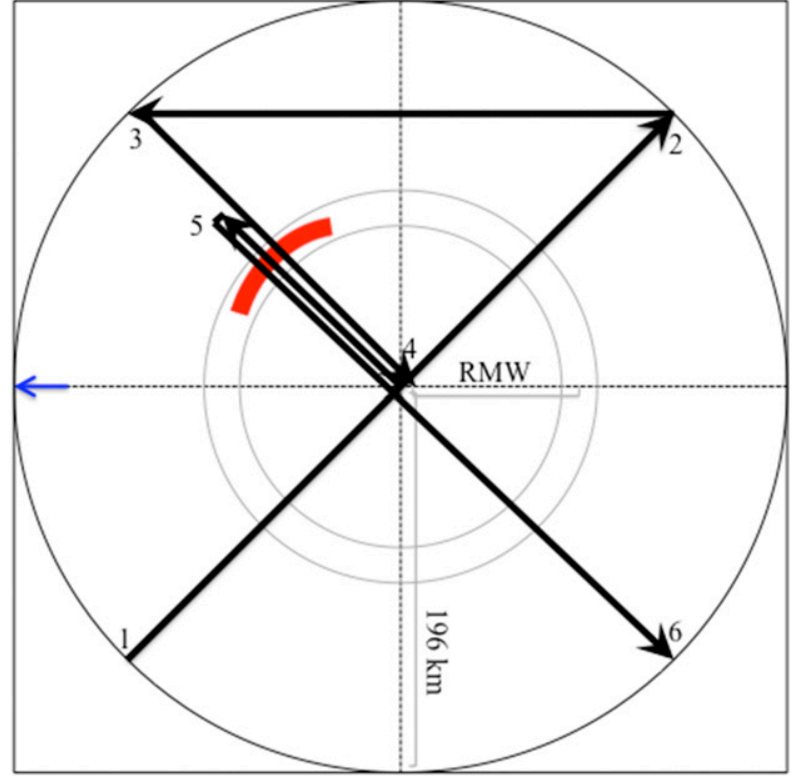

FIG. 7. A schematic diagram of the resampled figure-four pattern is shown. The blue arrow indicates the direction of storm motion, and the red curve signifies the traditional location of the peak wind speed.

simulated storms. These averages are computed over the full duration of the simulation period designated previously. For the single figure-four results, the 10 -min mean underestimate is within $1 \%$ of the 1 -min mean value for storms that have a higher frequency of peak wind speeds $<60 \mathrm{~m} \mathrm{~s}^{-1}$. The 10 -min mean values are mostly impacted above this wind speed threshold; therefore, Hurricane Bill and Ideal5 have a much lower underestimate overall, compared to the 1-min mean. The underestimate of the 6-h mean relative to the 1-min value is slightly larger. Comparing the 1-min mean values among all simulations for the single figure-four pattern also indicates that there is a connection between the strength and organization of the storm and the mean underestimate. The results from Bill, compared to
HNR2, quantify this idea in that a weak and disorganized storm has a much higher underestimate $(16.1 \%)$ than a storm that is strong and well organized (4.8\%).

The single butterfly results in Table 2 indicate similar trends among the three time-averaging methods as seen with the single figure-four. Because this flight pattern samples the hurricane eyewall one additional time, compared to the single figure-four, one might expect lower underestimates, compared to the single figure-four pattern. A lower underestimate indicates that the difference between the overall model peak and SFMR peak wind speed is reduced (i.e., percentages decrease), and an increase in the underestimate indicates a rise in this difference (i.e., percentages increase). The expectation of lower underestimates is mostly true, where the butterfly mean underestimates are between $0.2 \%$ and $1.0 \%$ lower for all model simulations, with the exception of Hurricane Bill. The additional passes through the maximum wind bands increase the underestimate by as much as $2.2 \%$ for the 6-h mean value. These differences are not statistically significant at $95 \%$ confidence.

\section{b. Repeated and rotated figure-four}

The results from the longer-duration flight patterns, which include the repeated and rotated figure-four, are also provided in Table 2. For the rotated figure-four flights, there is less of a difference among underestimates across the five simulated storms, compared to the single figure-four results. Looking at the 1-min mean winds, the single figure-four flights have a difference of $11.3 \%$ between HNR2 and Hurricane Bill, while the rotated figure-four has a difference of $8.8 \%$. The conjecture here is that by sampling more of the storm, it is possible to obtain SFMR-like wind speeds closer to the peak value. This idea holds true when evaluating the repeated figure-four results because there is less coverage of the storm, and the largest difference across all storms is $1.4 \%$ higher than the rotated figure-four overall. The underestimates for the rotated figure-four

TABLE 1. The number of flights is listed as a function of simulated storm. Also included is the total flight time (in h) for each type of pattern within each model. Flight times for one flight in each of the noted sampling patterns are 2.47 (single figure-four), 3.43 (single butterfly), 5.58 (repeated figure-four), 5.28 (rotated figure-four), and $3.0 \mathrm{~h}$ (resampled figure-four).

\begin{tabular}{|c|c|c|c|c|c|c|c|}
\hline & \multirow[b]{2}{*}{ No. of flights } & \multicolumn{6}{|c|}{ Total simulated flight time (h) } \\
\hline & & $\begin{array}{c}\text { Single } \\
\text { figure-four }\end{array}$ & $\begin{array}{c}\text { Single } \\
\text { butterfly }\end{array}$ & $\begin{array}{l}\text { Repeated } \\
\text { figure-four }\end{array}$ & $\begin{array}{l}\text { Rotated } \\
\text { figure-four }\end{array}$ & $\begin{array}{l}\text { Resampled } \\
\text { figure-four }\end{array}$ & Total \\
\hline HNR1 & 584 & 1442.5 & 2003.1 & 3258.7 & 3083.5 & 1752.0 & 11539.8 \\
\hline HNR2 & 88 & 217.4 & 301.8 & 491.0 & 464.6 & 264.0 & 1738.8 \\
\hline Bill & 232 & 573.0 & 795.8 & 1294.6 & 1225.0 & 696.0 & 4584.4 \\
\hline Ideal: Cat 5 & 648 & 1600.6 & 2222.6 & 3615.8 & 3421.4 & 1944.0 & 12804.4 \\
\hline Ideal: Cat 3 & 648 & 1600.6 & 2222.6 & 3615.8 & 3421.4 & 1944.0 & 12804.4 \\
\hline Total & 2200 & 5434.1 & 7545.9 & 12275.9 & 11615.9 & 6600.0 & 43471.8 \\
\hline
\end{tabular}




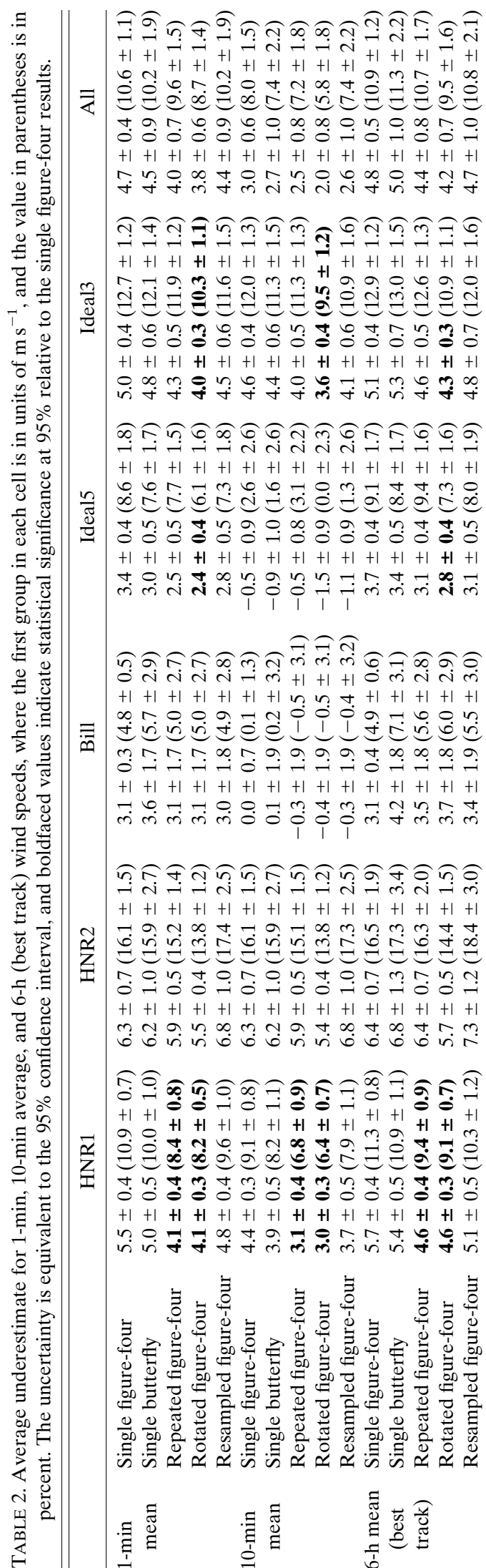

in HNR1, Ideal5, and Ideal3 are statistically significantly lower (at 95\% confidence) than single figure-four underestimates. The same significance metric is only true for the repeated figure-four within HNR1. This result suggests that sampling over a variety of storm intensities and increasing azimuthal coverage results in a reduced underestimate from SFMR overall.

\section{c. Resampled single figure-four}

Occasionally, it is not possible to conduct a repeated or rotated figure-four pattern due to time constraints. An interesting compromise is presented here to sample the maximum wind region more than a typical single figure-four pattern while only adding about $30 \mathrm{~min}$ to the flight. As shown in Table 2 for the 1-min mean values, the underestimate from the resampled figure-four is $1 \%-1.5 \%$ lower than the single figure-four for HNR1 and the two idealized simulations. Hurricane Bill shows a negligible difference between the patterns, while HNR2 is degraded by $1.3 \%$, compared to the single figure-four. Similar trends are found with the other time-averaging periods. Because the storm in HNR2 is disorganized for a long portion of the simulation, the results here suggest that the resampled figurefour pattern is not useful for the early stage tropical cyclogenesis or for storms with poorly defined wind structures. The slight improvement overall shows the resampled figure-four could serve as a useful alternative option for well-defined TCs in time-constrained situations if longer-duration patterns are not possible.

\section{d. Life cycle stages}

Single-storm averages discussed above are useful for pointing out differences in pattern types, but it is not clear how much they vary in terms of changes between different TC life cycle stages. Figure 8 shows the time series of wind speeds for HNR1 and the mean 1-min underestimate values with associated $95 \%$ confidence intervals at each flight time period for the single figurefour pattern. Portions of the time series are separated into five stages of TC evolution: tropical storm (stage I), rapid intensification (stage II), recently intensified (stage III), steady-state mature (stage IV), and recurving and weakening (stage V). The mean SFMR peak wind speeds never exceed the model maximum, but several time periods have their minimum underestimate (green triangle) within close proximity to the 6-h best track mean model wind speed. Table 3 provides a brief description of each stage and periods used from HNR1 and Ideal5. Figure 9 shows box-and-whisker plots of these five life cycle stages using data from HNR1 and Ideal5 and for the five complete simulations previously presented. The left panel is representative of the results in Table 2. 
It is worth mentioning the noticeable influence of storm size and structure on the likelihood of observing peak wind speeds from SFMR. The narrower range of the Hurricane Bill and HNR2 underestimates is a consequence of the relatively constant storm types depicted in their limited simulation periods. For the other three simulations that sample more of the TC life cycle, the interquartile range is much larger due to the changes in size and structure throughout each simulation.

Stage I has a higher median underestimate than stages II-V for both single and rotated figure-four patterns. The lowest underestimates occur in stages II and III, where the storm is beginning to contract and intensify to a small RMW intense TC (see Fig. 5). The largest differences between the single and rotated figure-four patterns occur in stages I and II. At these points in the model simulations, the TCs experience large changes in storm structure, and the rotated figure-four is able to capture those changes better than the single figure-four. Despite the higher variability in the rotated figure-four in stages I and II, its median underestimate is $3.5 \%-4 \%$ less than the single figure-four pattern. In stages III-V, there is less variability in the underestimate, as indicated by the interquartile ranges, but the median underestimates increase as the storm gets larger. In these final three stages, the median of the rotated figure-four pattern is $\sim 2 \%$ less than the single figure-four. These results convey that the rotated figure-four pattern will give a better estimate of the peak wind and significantly improves the underestimate during intensification periods.

Figure 10 provides similar information to Fig. 9 but uses the 10-min mean underestimate values. In the different life cycle stages, the underestimates are $>5 \%$ lower for stages III and IV, compared to the 1-min average for single figure-four flights. The difference for rotated figure-four flights is closer to $5 \%-7 \%$ lower when comparing the 10 -min to the 1 -min average. The overall result is that underestimates are reduced for 10-min winds, but the trends are similar to those for the 1-min winds, where underestimates are lowest in stages II and III before increasing again in stages IV and V.

Similarly to Figs. 9 and 10, Fig. 11 displays the box-andwhisker plots for the 6-h mean underestimates. The results for the different simulations are similar to the 1-min results in Fig. 9, with nearly identical interquartile ranges. However, there are some significant differences within the life cycle stages between the two flight patterns and with the 1-min mean results. For the flight pattern differences, stage II has lower median underestimates by $3.1 \%$, which is the largest difference of the five stages.
The other four stages have median differences of $\sim 1.5 \%$. When comparing against the 1-min averaging method, stages I and II have the most significant differences. The interquartile range for these periods is between $2 \%$ and $3 \%$ larger for the $6-\mathrm{h}$ mean of the single figure-four pattern with median values more than $1 \%$ higher for the 6 -h average. Stages III-V have differences of $\sim 0.5 \%$ or less between the averaging methods. These stark contrasts are a result of the structural changes occurring in stages I and II, where more variability in the peak model wind speeds occurs in the 6-h average and causes an increase in the underestimate.

\section{e. Correlation to storm size and asymmetric structure}

The results of the various simulations and periods of development confirm that size and intensity are closely linked to the underestimate. A question remains as to which structure-related parameters would be most useful for providing guidance during storm intensity assessment periods. To obtain this information, six variables related to storm intensity, size, and organization are correlated to the peak model wind speed. Figure 12 provides these correlations for the RMW near the surface and above the boundary layer (10-m and $2-\mathrm{km}$ heights), for the asymmetry parameter, for the peak azimuthal-mean tangential wind speed near the surface and above the boundary layer, and for the peak wind speed near the surface. These calculations are performed on the combined data of all simulations in this study.

The asymmetry parameter (Fig. 12c) has a significantly lower correlation with peak wind speed than all other variables. The highest correlation occurs with the peak SFMR wind speed (Fig. 12f), which is expected based on the results in the previous subsections. Tangential wind speeds near the surface are also highly correlated (Fig. 12e), but because the SFMR only provides wind speed and not a wind vector, it is difficult to obtain the tangential winds in an operational situation. The near-surface RMW (Fig. 12b) is highly correlated to the underestimate as well, and this result demonstrates the need to include storm size in the assessment. The reason the RMW and tangential wind speed above the boundary layer are provided is to show that in the absence of SFMR or surface wind data, it is still possible to obtain estimates about the underestimate. While these two parameters are not discussed further, it is possible to compute tangential wind speed at this altitude on the operational aircraft because the wind data are provided in vector form. Nonetheless, the near-surface peak SFMR wind speed and RMW are the most useful for relating storm structure and intensity to the peak wind underestimate. 


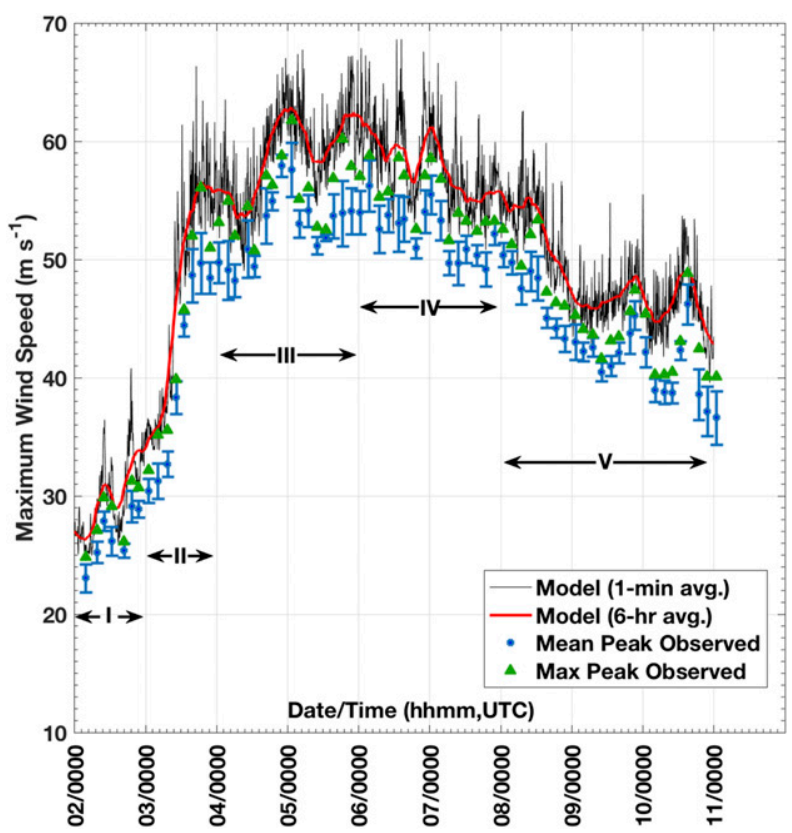

FIG. 8. The time series of HNR1 for the time period indicated in Fig. 3 is shown. The thin, black line indicates the maximum 1-min-average 10-m wind speed from the model field, and the red line indicates the 6-h average from the model, similar to a best track estimate. The blue markers are the average maximum surface wind speed obtained from the eight simulated single figure-four flight tracks with the error bars indicating the $95 \%$ confidence intervals. The green marker is the maximum aircraft wind speed obtained within the simulated flight time window. The groups labeled with I, II, III, IV, and V correspond to the disorganized, RI, smallmature, large and steady, and recurving phases of the TC life cycle.

\section{Applications and comparisons to real cases}

The results of this study so far convey that multiple factors affect undersampling of the peak surface wind speed by hurricane hunter aircraft. The most significant are storm size, as determined by RMW, and the maximum surface-measured wind speed. Development of a correction technique based on the storm size and intensity is the most logical method to provide guidance on the underestimate but requires some knowledge of the current storm intensity. For the simulated flights, the peak wind speed within the full field is known, making it simpler to develop a new term called an undersampling rate, $R_{U}$. For example, a 6-h mean wind speed of $100 \mathrm{kt}$ associated with an SFMR peak wind speed of $95 \mathrm{kt}$ suggests an undersampling rate of $5 \%$. However, in operational practice, the definition of the undersampling rate must be inverted because the peak wind speed within the wind field is unknown. We define a correction factor $C_{U}$ as a function of the undersampling rates $R_{U}$ computed in this study:

$$
C_{U}=\left(\frac{10^{4}}{100-R_{U}}\right)-100
$$

where $C_{U}$ and $R_{U}$ are in percent. From the example above, Eq. (4) computes a correction factor of $5.3 \%$ instead of the $5 \%$ suggested by the undersampling rate. However, the differences become larger as $R_{U}$ increases (e.g., for $R_{U}=15 \%, C_{U}=17.6 \%$ ). Table 4 provides the correction factors for all flight patterns, with the exception of the resampled figure-four, associated with the 6-h best track time average as a function of storm size and intensity. Table 4 indicates that for a large tropical storm, the best-case scenario is a $16 \%$ correction when flying the rotated figure-four pattern. A single figure-four pattern requires a $19 \%$ increase. For a small major hurricane, all flight patterns would require $<2 \%$ increase in the peak wind speed. These low corrections are found in the small, nonmajor hurricanes as well. The remaining combinations fall somewhere between these extremes. An intensity estimate is necessary to determine $R_{U}$, and for historical cases, the best track value is available for this purpose. In real-time situations, a best track intensity may not be determined at the time of the flight, and the recommendation here is to treat the SFMR peak wind speed during a flight as the best estimate of the best track equivalent peak wind speed. The correction factors in Table 4 could then be applied to this value. Given that the correction factors have a variability of $\pm 2 \%-3 \%$ overall with additional variability on the order of $1 \%-2 \%$ from the best track averaging methods, these values are meant to stand as guidelines to be applied at the discretion of the user.

Examining real observations will help visualize how these corrections could be applied. One example is taken from the 2 September NOAA P-3 mission into Hurricane Earl during the 2010 Atlantic hurricane season. Figure 13 provides the flight track from the NOAA P-3 mission overlaid on the best track position and precipitation coverage from the lower fuselage radar (Marks 1985). Radial profiles of SFMR wind speeds are provided as well. The observed peak wind speed from SFMR during the flight was $87 \mathrm{kt}$, and the best track intensity was $90 \mathrm{kt}$. The undersampling rate and subsequent correction factor are $3.3 \%$ and $3.4 \%$, respectively. A rotated figure-four pattern was flown for this category 2 hurricane with RMW of $27.5 \mathrm{n} \mathrm{mi}$ $(51 \mathrm{~km})$, and Table 4 suggests a correction factor of $8 \%$. Applying this correction factor to the SFMR peak wind speed yields a new intensity estimate of $94 \pm 3 \mathrm{kt}$. Airborne data are often given more weight in the best track assessment; therefore, it is not surprising that the best track and maximum SFMR wind speed are similar here for that reason. The SFMR underestimates the peak 1-min wind speed at varying magnitudes, and in conjunction, best track estimates dependent on SFMR 
TABLE 3. A brief description of the characteristics of each life cycle stage is provided along with the portion of HNR1 and Ideal5 used for the statistical evaluation of the peak wind speed underestimate.

\begin{tabular}{|c|c|c|}
\hline Stage & Description & Model period \\
\hline I & $\begin{array}{l}\text { Tropical storm: Large RMW and wind } \\
\text { speeds }<33 \mathrm{~m} \mathrm{~s}^{-1}\end{array}$ & $\begin{array}{l}\text { HNR1: } 0000 \text { UTC } 2 \text { Aug-0000 UTC } 3 \text { Aug } \\
\text { Ideal5: } 0000 \text { UTC } 1 \text { Sep-0600 UTC } 3 \text { Sep }\end{array}$ \\
\hline II & $\begin{array}{l}\text { Rapid Intensification: Change in intensity } \\
\text { by } 15 \mathrm{~m} \mathrm{~s}^{-1} \text { in a } 24 \mathrm{~h} \text { period, RMW } \\
\text { decreasing }\end{array}$ & $\begin{array}{l}\text { HNR1: } 0000 \text { UTC } 3 \text { Aug-0000 UTC } 4 \text { Aug } \\
\text { Ideal5: } 0600 \text { UTC } 3 \text { Sep-0000 UTC } 5 \text { Sep }\end{array}$ \\
\hline III & $\begin{array}{l}\text { Recently Intensified: Slowing to steady } \\
\text { intensification with intense peak winds } \\
\left(>55 \mathrm{~m} \mathrm{~s}^{-1}\right) \text { and RMW }<50 \mathrm{~km}, \\
\text { after rapid intensification }\end{array}$ & $\begin{array}{l}\text { HNR1: } 0000 \text { UTC } 4 \text { Aug-0000 UTC } 6 \text { Aug } \\
\text { Ideal5: } 0000 \text { UTC } 5 \text { Sep-0000 UTC } 7 \text { Sep }\end{array}$ \\
\hline IV & $\begin{array}{l}\text { Steady state mature: Steady } \\
\text { intensification rate }\left( \pm 5 \mathrm{~m} \mathrm{~s}^{-1}\right) \text { and } \\
\text { little change or slight increases in } \\
\text { RMW, winds }>50 \mathrm{~m} \mathrm{~s}^{-1}\end{array}$ & $\begin{array}{l}\text { HNR1: } 0000 \text { UTC } 6 \text { Aug-0000 UTC } 8 \text { Aug } \\
\text { Ideal5: } 0000 \text { UTC } 7 \text { Sep-0000 UTC } 9 \text { Sep }\end{array}$ \\
\hline $\mathrm{V}$ & $\begin{array}{l}\text { Recurving and weakening: Continuous } \\
\text { intensity decrease with increasing } \\
\text { RMW, or track turning north to } \\
\text { northeast }\end{array}$ & $\begin{array}{l}\text { HNR1: } 0000 \text { UTC } 8 \text { Aug-0000 UTC } 11 \text { Aug } \\
\text { Ideal5: } 0000 \text { UTC } 9 \text { Sep-0000 UTC } 11 \text { Sep }\end{array}$ \\
\hline
\end{tabular}

will likely underestimate the unobserved TC maximum 1-min wind (full field). The uncertainty of the best track intensity accounts for some of the low estimate relative to the corrected intensity of $94 \mathrm{kt}$, but a more useful method might be to incorporate knowledge of the SFMR underestimate into the formulation of the best track value, as the Hurricane Earl example suggests.

It is possible to perform a statistical analysis of this metric using the full set of real cases mentioned in section $2 \mathrm{c}$. For each case, the flight pattern, RMW, and intensity are obtained, and the underestimate correction is assigned. Figure 14 provides joint histograms of the peak wind speed difference as a function of best track intensity. The results effectively show the histogram of the peak wind speed difference for each best track bin (every $15 \mathrm{kt}$ ), where the maximum probability is denoted with the black marker and $95 \%$ confidence interval. Note that there are few cases in this sample data that have peak wind speeds $>135 \mathrm{kt}$, and the results within that bin are not as meaningful. For this analysis, the best track intensity is treated as an estimate of the wind speed for the full wind field despite the fact that in reality it is not. In Fig. 14a, the difference in peak wind speed (i.e., $\Delta V_{\max }=V_{\text {max,BT }}-$ $\left.V_{\text {max,sfmr }}\right)$ is generally in the range of $0-10 \mathrm{kt}$, with the
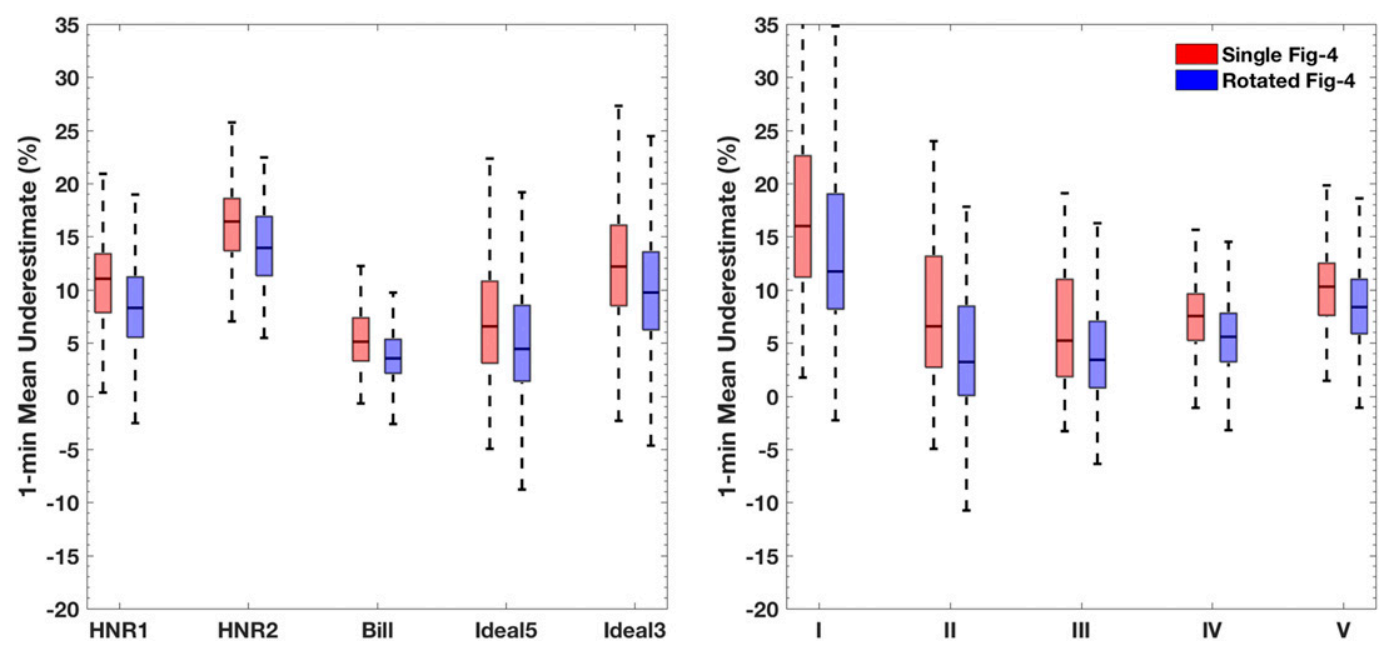

FIG. 9. (left) Box-and-whisker plots of the 1-min mean underestimate (\%) are provided for single (red) and rotated (blue) figure-four flight patterns. (right) As on the left, but for the five life cycle periods mentioned in the text from HNR1 (Fig. 8) and Ideal5. The boxes include the interquartile range, and the dashed lines indicate the maximum and minimum underestimates for each sample. 

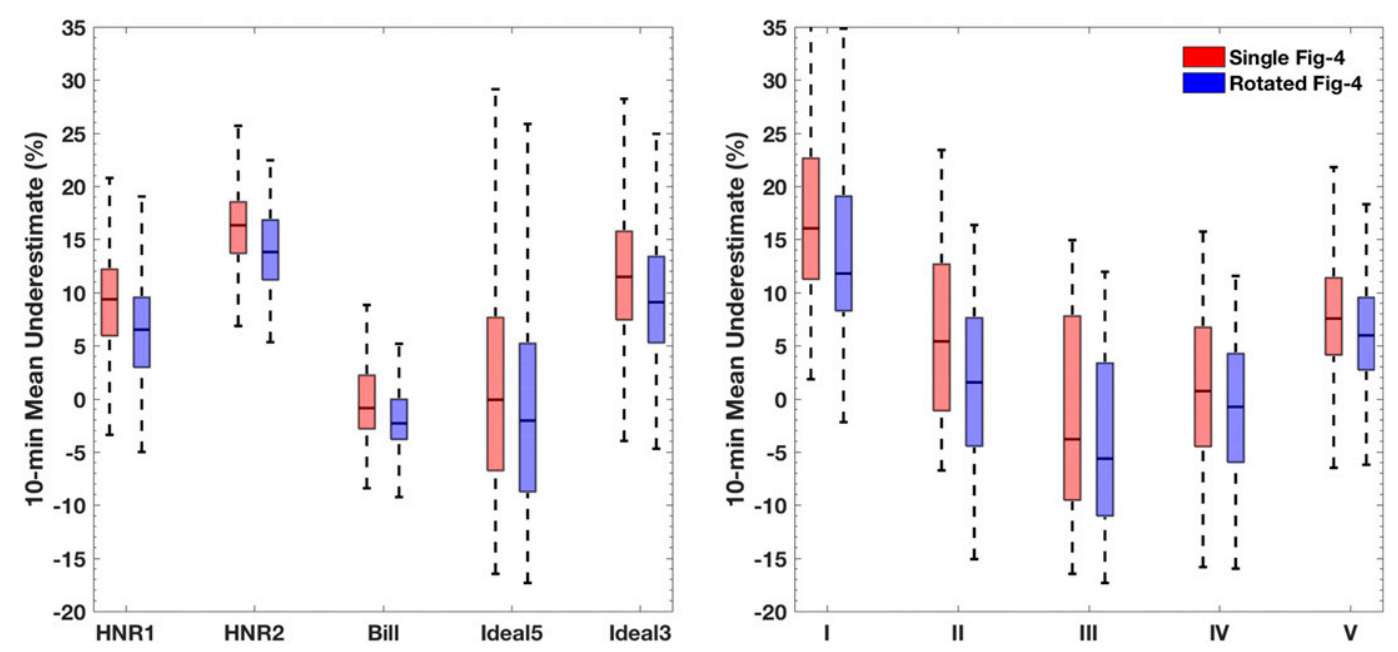

FIG. 10. As in Fig. 9, but for 10-min mean underestimates.

difference increasing at the higher wind speeds. The probabilities here confirm that standard SFMR peak wind speeds do not fully capture the estimated 1-min peak wind speed. Figure 14b shows the same histograms, but with the correction factor applied to the peak SFMR wind speeds. These histograms indicate that the SFMR is closer to the peak overall wind speed from best track as the differences range from -5 to $5 \mathrm{kt}$ for most intensity bins. Negative values indicate that the peak estimate from best track is low relative to the expected peak wind speed determined from the correction factor. It may seem unexpected that the highest probabilities of the adjusted peak wind speed are very similar to the best track estimates in Fig. 14b. There is an increased percentage of cases in these wind speed bins with the corrected value exceeding the best track, suggesting that the best track estimate is too low. It is also important to indicate the best track values used are a result of a blending of SFMR, flight-level reduction, and dropsonde wind speeds, which take into account some of the undersampling (Franklin et al. 2003). The peak wind evaluation may be somewhat affected by these other factors, but the main conclusion here is that applying the correction factors in Table 4 to SFMR peak wind speeds will promote improved representation of the expected peak wind speed (full wind field) in the best track and reduce the uncertainty from undersampling.

\section{Conclusions}

Aircraft data collected in tropical storms and hurricanes are important for a variety of reasons, and as has
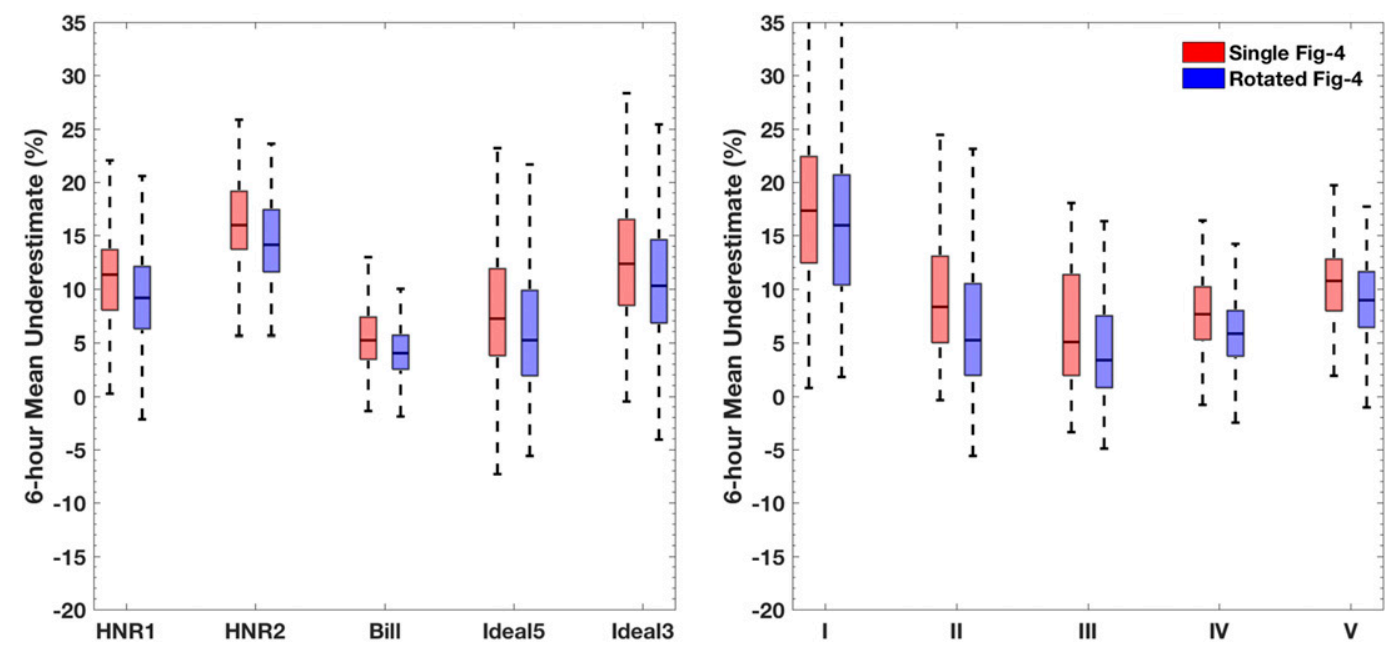

FIG. 11. As in Fig. 9, but for the 6-h mean wind speed. 



FIG. 12. Scatterplots of the 1-min, 10-m maximum wind speed underestimate ( $V_{\text {max }, 10 \mathrm{~m}}$; in percent) relative to the (a) 2-km RMW $\left(\mathrm{RMW}_{z=2 \mathrm{~km}}\right)$, (b) 10-m RMW (RMW ${ }_{z=10 \mathrm{~m}}$ ), (c) asymmetry ratio, (d) 2-km maximum tangential wind speed $\left(V_{t, z=2 \mathrm{~km}}\right),(\mathrm{e}) 10-\mathrm{m}$ maximum tangential wind speed $\left(V_{t, z=10 \mathrm{~m}}\right)$, and (f) 10-m maximum wind speed $\left(V_{z=10 \mathrm{~m}}\right)$. The linear regression (solid) and $95 \%$ confidence (dashed) lines are provided along with the correlation coefficient in each panel.

been reinforced in this study, they can significantly improve the ability to make decisions about the strength of a storm. Since its operational inception, the SFMR has been a key part of the operational data suite, and it is well known that SFMR cannot sample the actual peak $10-\mathrm{m}$ wind speed. Within the SFMR OSSE framework described, this study confirms that an underestimate in the peak wind speed is expected but goes several steps further in conveying when and by how much to apply a correction. The two main goals of this study were to quantify the undersampling by SFMR through the different TC life cycle stages and to develop a technique to apply a correction to the TC intensity estimate based on this knowledge. The results and discussion are therefore summarized into several main conclusions:

- Using a WRF Model framework that is known to produce realistic hurricane wind fields, it was determined that the simulated SFMR wind speed undersampling is highly correlated with the storm size and intensity, where large tropical storms have the highest underestimates $(>14 \%)$, and small intense hurricanes have the lowest underestimates $(<4 \%)$. These underestimates are also reflective of the different TC development stages.

TABLE 4. Average percent underestimate against the 6-h maximum wind speed for storm intensity and size, which are defined by the Saffir-Simpson hurricane wind scale and near-surface RMW speed, respectively. Storm strength is binned according to the peak SFMRlike wind speeds. The values are provided for the single figure-four, single butterfly, repeated figure-four, and rotated figure-four patterns.

\begin{tabular}{lcrr}
\hline \hline \multicolumn{1}{c}{ Size/category } & Tropical storm & Categories $1-2$ & Categories 3-5 \\
\hline Small RMW $<15 \mathrm{n} \mathrm{mi}$ & $10 \%, 14 \%, 10 \%, 9 \%$ & $5 \%, 3 \%, 0 \%, 1 \%$ & $2 \%, 1 \%, 0 \%, 0 \%$ \\
Medium $15<$ RMW $<30 \mathrm{n} \mathrm{mi}$ & $15 \%, 16 \%, 14 \%, 12 \%$ & $9 \%, 9 \%, 8 \%, 8 \%$ & $5 \%, 4 \%, 4 \%, 4 \%$ \\
Large RMW $>30 \mathrm{n} \mathrm{mi}$ & $19 \%, 18 \%, 17 \%, 16 \%$ & $11 \%, 10 \%, 9 \%, 9 \%$ & $8 \%, 7 \%, 6 \%, 6 \%$ \\
\hline
\end{tabular}





FIG. 13. (left) The path of the NOAA P-3 flight from $\sim 0900$ to 1430 UTC 2 Sep 2010 into Hurricane Earl. The light gray line indicates the best track positions, and the shaded contours indicate the lower fuselage C-band radar reflectivity $(\mathrm{dBZ})$ from the first center position determined during the mission (black circle; 2203 UTC). (right) The SFMR wind speed radial profiles for each pass, where the initial point is on the southwest side of the hurricane. Negative radii indicate the inbound portions of the full radial pass.

- Comparing different flight patterns indicates that increasing the number of radial passes significantly improves the SFMR peak wind underestimate on the order of $2 \%-3 \%$ overall, and the increased sampling is especially useful during TC intensification and TC maturation, where underestimates improve by $>4 \%$.

- Analysis of real storm SFMR peak wind speeds and best track intensities shows that not incorporating the undersampling principles results in a 5-10-kt peak wind underestimate, but applying the correction factor to the SFMR winds improves this underestimate by at least $5 \mathrm{kt}$ at most storm intensities. By first accounting for the underestimate in the SFMR peak wind and using the correction factor, best track estimates relying on SFMR winds will be more representative of the peak wind speed within the entire TC wind field.
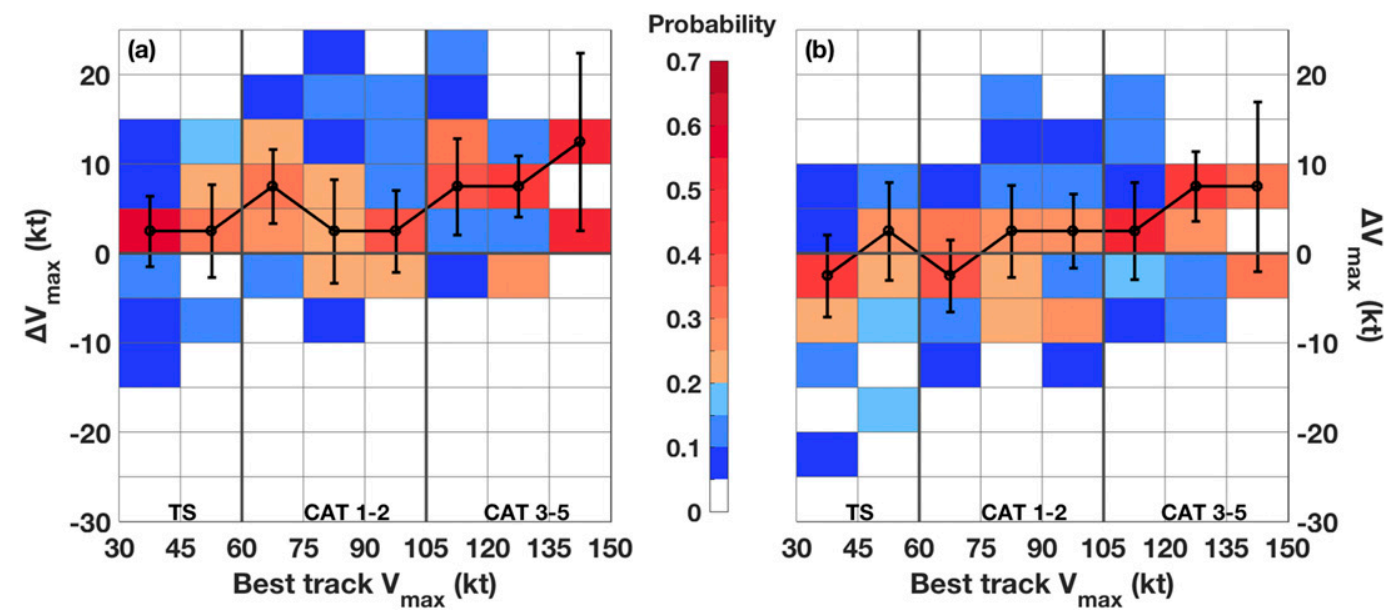

FIG. 14. Joint histograms of the difference between the best track intensity and SFMR peak wind speed $\left(\Delta V_{\max }\right)$ are provided as a function of best track intensity for (a) original SFMR winds and (b) undersampling-corrected SFMR winds. The differences are reported as probability within each best track intensity bin. The black line indicates the peak probability for each best track bin, with error bars indicating the $95 \%$ confidence interval. 
The guidelines of the correction factor are meant to serve as reference for the tropical cyclone community when assessing the intensity based on aircraft SFMR wind speeds. The methodology and results in this study suggest one path to obtain better estimates of storm intensity, but other data sources could help in this regard. One topic of ongoing work is related to assessing intensity using scatterometer winds, which would be most useful at tropical storm to minimal hurricane wind speeds. The minimum central pressure is another avenue for diagnosing intensity, and some analysis of simulated dropsondes in the eyes of hurricanes indicates a potential influence on representing the intensity. These two topics will be discussed in future studies. The overarching problem here still remains in that a point intensity estimate does not convey storm structure characteristics. It could be useful in the future to utilize alternative intensity metrics or structural analyses, such as described by Vukicevic et al. (2014) and Klotz and Jiang (2017), to account for the full wind field variability and its relationship to TC strength.

Acknowledgments. We thank the United States Weather Research Program and the Joint Hurricane Testbed (JHT) at the National Hurricane Center for supporting this work (Grant NA15OAR4590203). Special thanks are given to the JHT committee members for their comments and guidance throughout the project. Dr. Yumin Moon produced the updated simulation of Hurricane Bill (2009). We also want to thank Dr. Eric Uhlhorn for providing clear guidance on simulating SFMR wind speeds and helping us initiate this work. Additionally, we would like to express gratitude to the two internal reviewers at NOAA's Atlantic Oceanographic and Meteorological Laboratory for their helpful advice regarding this work. Several additional reviewer comments also significantly aided our final product.

\section{APPENDIX A}

\section{Model Simulation Details}

The first two simulations in our set of five are hurricane nature run 1 (Nolan et al. 2013) and hurricane nature run 2 (Nolan and Mattocks 2014). These are both regionally downscaled simulations of hurricanes produced in the Atlantic by the joint OSSE nature run (JONR; Andersson and Masutani 2010). The two simulations use the same grid spacing, vertical levels, and physical parameterizations, and both simulate the complete evolution of Atlantic hurricanes from tropical disturbance to recurving cyclone. HNR1 was produced with version 3.2.1 of WRF, while HNR2 was produced with version 3.4.1.

HNR1 provides 13 days of model output from its 1-km domain at 6-min intervals. Because of land interactions, only about $36 \mathrm{~h}$ of HNR2 (saved at 5-min intervals) are usable for this study. To increase sample size, the Hurricane Bill (2009) simulation of Moon and Nolan (2015) was also repeated, using WRF 3.4.1 and again using the same resolution, model levels, and parameterizations. This simulation provides 3.5 days of $1-\mathrm{km}$ output at 5 -min intervals. However, the structure and intensity of Bill do not change much during the simulation.

To further expand the dataset, two entirely new simulations were produced using the idealized modeling framework of Nolan (2011). The model domain is a large zonally periodic channel, initialized with a purely zonal flow that varies with height. The flow is balanced with meridional gradients of pressure and temperature that are computed from an iterative scheme [see the appendix of Nolan (2011)]. The SST, initial vortex, mean flow, and wind shear were selected so as to produce two different TCs: one that rapidly intensifies to a fairly small category 5 hurricane (Ideal5) and one that develops more slowly into a larger and more asymmetric category 3 hurricane (Ideal3).

The Ideal5 and Ideal 3 simulations use an outer domain with $240 \times 180$ grid points and vortex-following nested grids of $240 \times 180,180 \times 180,360 \times 360$, and $480 \times 480$ grid points, with 27-, 9-, 3-, and 1-km grid spacings, respectively. Both use 60 vertical levels between the surface and $20-\mathrm{km}$ height, with the same vertical grid spacings as in the hurricane nature runs [see Fig. 2 of Nolan et al. (2013)]. The physical parameterizations are also the same, including the use of the onedimensional mixed-layer cooling scheme of Pollard et al. (1972) available with WRF 3.4.1. All simulations used time steps of $60,30,10$, and $5 \mathrm{~s}$ on their 27-, 9-, 3-, and 1-km grids, respectively, and the important properties of the five simulations are summarized in Table A1.

Ideal5 is initialized with $5 \mathrm{~m} \mathrm{~s}^{-1}$ of easterly flow from the surface up to $850 \mathrm{hPa}$, then transitioning smoothly to $0 \mathrm{~m} \mathrm{~s}^{-1}$ between 850 and $200 \mathrm{hPa}$ (i.e., $5 \mathrm{~m} \mathrm{~s}^{-1}$ of westerly wind shear). The Coriolis parameter is $f=3.77 \times$ $10^{-5} \mathrm{~s}^{-1}$ (corresponding to $15^{\circ} \mathrm{N}$ latitude, although $f$ does not vary across the channel). The environmental sounding at the central latitude of the domain is the Dunion (2011) moist tropical sounding; this sounding varies meridionally due to the geostrophic wind balance of the shear, but the specific humidity is recomputed to keep the relative humidity profile constant. The SST is set to $29^{\circ} \mathrm{C}$ along the centerline of the domain, but also varies meridionally. This meridional variation matches the temperature variation that exists at $5-\mathrm{km}$ altitude due to the thermal wind balance. Nolan (2011) used this modification to suppress excessive convection in the northern part of the channel, where the temperature difference between the SST and the midtroposphere 
TABLE A1. The basic properties related to domain, grid spacing, output frequency, vortex type, and forcing mechanism are summarized for the five WRF hurricane simulations used in this study.

\begin{tabular}{|c|c|c|c|c|c|}
\hline $\begin{array}{l}\text { Simulation } \\
\text { name }\end{array}$ & Domain & Grid sizes & $\begin{array}{l}\text { Output frequency } \\
\text { and duration }\end{array}$ & $\begin{array}{l}\text { Initial } \\
\text { vortex }\end{array}$ & Forcing \\
\hline HNR1 & Tropical Atlantic & $\begin{array}{l}27 \mathrm{~km}: 240 \times 160 \\
9 \mathrm{~km}: 120 \times 120 \\
3 \mathrm{~km}: 240 \times 240 \\
1 \mathrm{~km}: 480 \times 480\end{array}$ & $6 \mathrm{~min}$ for 13 days & $\begin{array}{l}\text { Realistic disturbance } \\
\text { from JONR }\end{array}$ & $\begin{array}{l}\text { JONR boundary conditions } \\
\text { and large-scale relaxation }\end{array}$ \\
\hline HNR2 & $\begin{array}{l}\text { Caribbean and } \\
\text { Gulf of Mexico }\end{array}$ & $\begin{array}{l}9 \mathrm{~km}: 480 \times 360 \\
3 \mathrm{~km}: 360 \times 360 \\
1 \mathrm{~km}: 480 \times 480\end{array}$ & $5 \min$ for 8 days & $\begin{array}{l}\text { Realistic disturbance } \\
\text { from JONR }\end{array}$ & $\begin{array}{l}\text { JONR boundary conditions } \\
\text { and large-scale relaxation }\end{array}$ \\
\hline Bill2.0 & West Atlantic & $\begin{array}{l}9 \mathrm{~km}: 384 \times 336 \\
3 \mathrm{~km}: 360 \times 360 \\
1 \mathrm{~km}: 480 \times 480\end{array}$ & $5 \mathrm{~min}$ for 3.5 days & $\begin{array}{l}\text { GFDL initialization of } \\
\text { Hurricane Bill }\end{array}$ & $\begin{array}{l}\text { GFDL analysis boundary } \\
\text { conditions }\end{array}$ \\
\hline Ideal5 & $\begin{array}{l}\text { Zonal channel with } \\
\qquad f=3.77 \times 10^{-5} \mathrm{~s}^{-1}\end{array}$ & $\begin{array}{l}27 \mathrm{~km}: 240 \times 180 \\
9 \mathrm{~km}: 180 \times 180 \\
3 \mathrm{~km}: 360 \times 360 \\
1 \mathrm{~km}: 480 \times 480\end{array}$ & 5 min for 10 days & $\begin{array}{l}10 \mathrm{~m} \mathrm{~s}^{-1} \text { midlevel } \\
\text { vortex }\end{array}$ & $\begin{array}{l}\text { Large-scale relaxation to } \\
\text { initial mean flow and shear }\end{array}$ \\
\hline Ideal3 & $\begin{array}{l}\text { Zonal channel with } \\
\qquad f=5.0 \times 10^{-5} \mathrm{~s}^{-1}\end{array}$ & $\begin{array}{l}27 \mathrm{~km}: 240 \times 180 \\
9 \mathrm{~km}: 180 \times 180 \\
3 \mathrm{~km}: 360 \times 360 \\
1 \mathrm{~km}: 480 \times 480\end{array}$ & 5 min for 10 days & $\begin{array}{l}15 \mathrm{~m} \mathrm{~s}^{-1} \text { low-level } \\
\text { vortex }\end{array}$ & $\begin{array}{l}\text { Large-scale relaxation to } \\
\text { initial mean flow and shear }\end{array}$ \\
\hline
\end{tabular}

would otherwise be substantially increased. The SST variation is approximately linear, ranging from $32.5^{\circ}$ to $25.5^{\circ} \mathrm{C}$ from the south to north edges of the channel. An initial vortex, along with the pressure and temperature anomalies required by gradient wind balance, is embedded into the flow at the eastern end of the domain. For Ideal5, the initial vortex has a maximum tangential wind of $10 \mathrm{~m} \mathrm{~s}^{-1}$ at a radius of $108 \mathrm{~km}$ and a height of $3.7 \mathrm{~km}$. The radial wind profile is a modified Rankine vortex with decay parameter $a=1 / 3$. The initial vertical structure of the wind field uses the same analytic function as the midlevel vortices in Eq. (4) of Nolan (2007). Following Nolan and McGauley (2012; see their Fig. 1.6), the midlevel humidity across the domain is also reduced by $10 \%$, which reduces rainband activity in the development stage and allows the vortex to intensify more rapidly and to a smaller size.

The Ideal3 simulation uses a central SST of $27^{\circ} \mathrm{C}$, $5 \mathrm{~m} \mathrm{~s}^{-1}$ of easterly flow, and $10 \mathrm{~m} \mathrm{~s}^{-1}$ of westerly shear, and it also uses the Dunion (2011) moist tropical sounding. Specific humidity is recomputed as above, but it is not reduced by $10 \%$ in the midlevels. The Coriolis parameter is set to $f=5.0 \times 10^{-5} \mathrm{~s}^{-1}$ (corresponding to $20^{\circ} \mathrm{N}$ ). The initial vortex has its maximum tangential wind of $15 \mathrm{~m} \mathrm{~s}^{-1}$ at radius $162 \mathrm{~km}$, again using a modified Rankine vortex with $a=1 / 3$, but with the wind maximized at a height of $1.5 \mathrm{~km}$ using the vertical structure formula of Stern and Nolan [2011; see their Eq. (4.2)].

As the Ideal5 and Ideal3 simulations evolve, the wind, temperature, and moisture fields on the outer domain are relaxed back to their initial values with a 24 -h relaxation time scale. This relaxation does not apply on the nested grids. The relaxation keeps the environmental sounding and wind shear profile around the cyclone roughly constant as the storm develops, but it does not interfere with the development and evolution of the storms.

Ideal5 and Ideal3 are both integrated for 10 days with surface fields on the $1-\mathrm{km}$ domain saved every $5 \mathrm{~min}$. In Ideal5, the storm develops quickly and begins RI at the start of day 3 , with peak surface wind speed exceeding $70 \mathrm{~m} \mathrm{~s}^{-1}$ from days 5 through 8 . The cyclone initially contracts to an RMW of about $18 \mathrm{~km}$ and then slowly increases in size. In Ideal3, the development is slower and more episodic, with the surface wind speed first exceeding $50 \mathrm{~m} \mathrm{~s}^{-1}$ on day 6 . For the first 7 days, the storm is much larger and much more asymmetric than Ideal5. After it becomes a hurricane, the RMW is $40 \mathrm{~km}$, but later it expands to $70 \mathrm{~km}$ with an eyewall replacement cycle. After this event, the storm then intensifies further, and the RMW contracts to $30 \mathrm{~km}$ at the end of the simulation.

\section{APPENDIX B}

\section{Sensitivity Assessment of Model Wind Fields}

To assess whether or not the spatial variability of the simulated wind fields is strongly dependent on the horizontal and vertical diffusion in the WRF simulations, Ideal5 and Ideal3 simulations were repeated with two modifications. In the first pair of modified simulations, the horizontal diffusion was increased by a factor of 4 everywhere (hereafter, Ideal5-KH4 and Ideal3-KH4). In the second pair, the Yonsei University boundary 

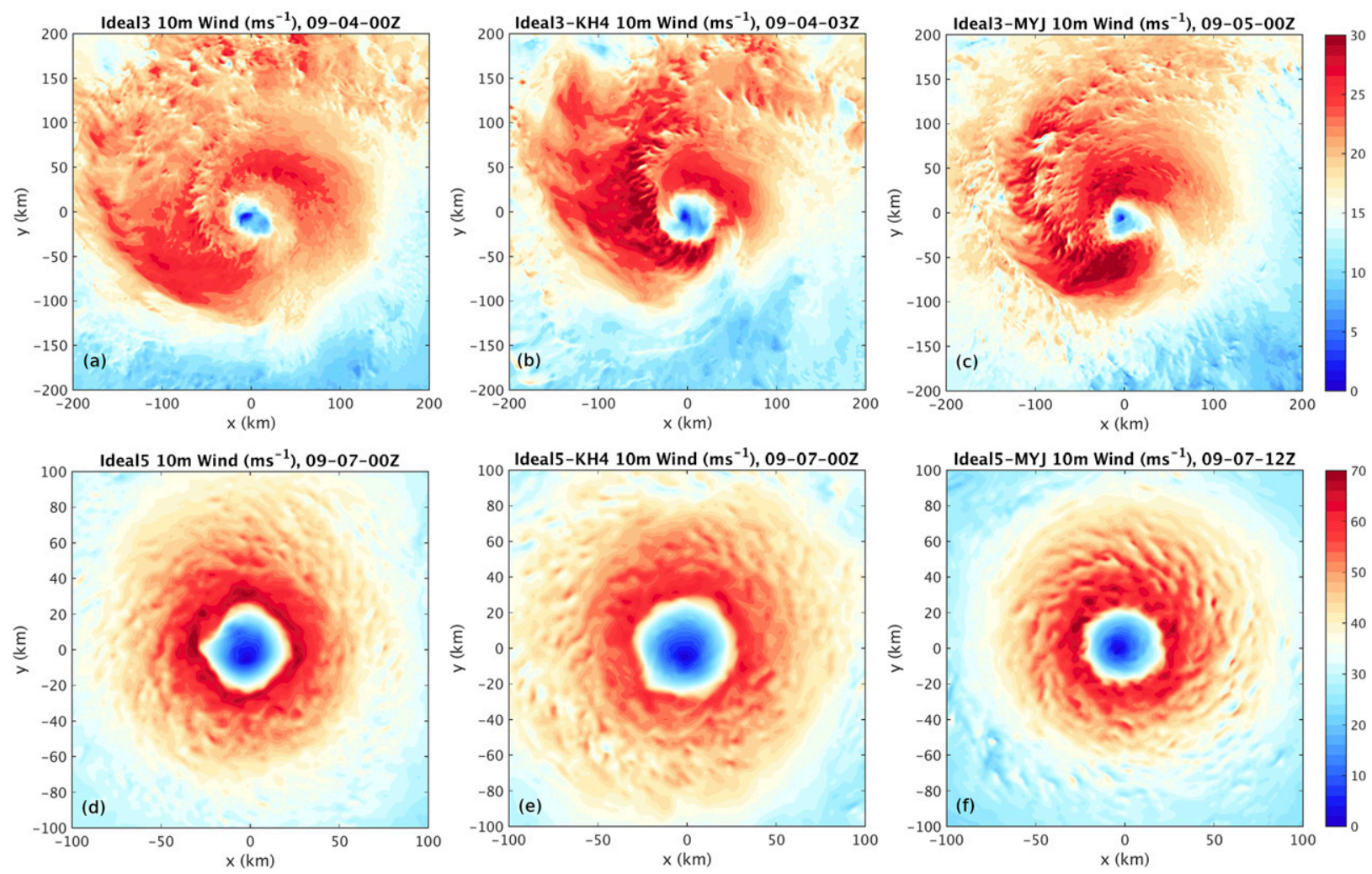

FIG. B1. Surface wind fields from the original and modified Ideal3 and Ideal5 simulations. (top) The wind field during the strong tropical storm stage of Ideal3 for the original, the increased horizontal diffusivity, and the MYJ scheme, respectively, at times when the wind fields were qualitatively similar. (bottom) The intense hurricane stage from Ideal5. The color scales and domain sizes are different for the two cases.

layer scheme (YSU; Hong et al. 2006) was replaced with the Mellor-Yamada-Janjić (MYJ; Janjić 1990, 1994) scheme (Ideal5-MYJ and Ideal3-MYJ). However, following the procedure of Nolan et al. (2009a,b), the wind speed-dependent formula for the surface roughness length over the ocean was replaced by the same formula as used in the YSU scheme, in which the surface roughness levels off to a constant value for wind speeds greater than $26 \mathrm{~m} \mathrm{~s}^{-1}$ (whereas the default formula continues to increase). For consistency with the hurricane nature run simulations, the wind speed formula from WRF version 3.1.1 was used.

These modifications caused minor changes to the evolutions of the simulated hurricanes. For both Ideal5 and Ideal3, increasing horizontal diffusion reduced the peak wind speeds and resulted in slightly larger eyewalls in the mature stage, but the timing of the intensification and the overall structural evolution was very similar. With the MYJ scheme, rapid intensification was delayed by about $24 \mathrm{~h}$ in both Ideal5 and Ideal3, but in both cases, the storm ultimately reached nearly identical intensity. The RMW was generally $5-10 \mathrm{~km}$ smaller for the storms using MYJ (not shown).

Figure B1 shows snapshots of the surface wind field from the original and modified simulations. Ideal 3 is used to show the wind field in a broad tropical storm, while Ideal5 is used to show the wind field of an intense hurricane. In each case, the output times have been selected subjectively so that the wind fields have similar structures. The tropical storm phase is illustrated in the top row. While a more quantitative analysis remains for future work, there do not appear to be significant differences in the three wind fields. At this stage, the eyewall has not yet formed, and the strongest surface winds are associated with bands of convection to the northeast and southeast of the center and in a broad area of convective cells to the north. Similar structures are apparent, with roughly the same length scales, in all three plots.

The intense hurricane phase is illustrated in the second row. The wind fields of all three simulations show the outward angled bands discussed in the text. However, the peak winds in all three storms are associated with more coherent features in the eyewall, and it is the spatial variability of these features that leads to undersampling by aircraft observations. Therefore, the wind structures outside the eyewall, which may or may not be realistic, do not contribute to the undersampling (which, in fact, is very small for these storms; see Table 4). 
To summarize, during the early stages, from developing tropical storm to category 1 hurricane, it is the convective cells embedded in rainbands and in the developing eyewall that produce localized wind maxima that have only a small chance of being intercepted by the aircraft. These wind structures do not appear to be strongly dependent on the horizontal and vertical diffusion. In the mature stage, undersampling is due to the wind variability in the eyewall, which is believed to be caused by dynamical instabilities that can be resolved by the model, but do not show significant differences in the modified simulations.

\section{REFERENCES}

Aberson, S. D., M. T. Montgomery, M. M. Bell, and M. Black, 2006: Hurricane Isabel (2003): New insights into the physics of intense storms. Part II: Extreme localized wind. Bull. Amer. Meteor. Soc., 87, 1349-1354, https://doi.org/10.1175/BAMS-87-10-1349.

_ A. Aksoy, K. J. Sellwood, T. Vukicevic, and X. Zhang, 2015: Assimilation of high-resolution tropical cyclone observations with an ensemble Kalman filter using HEDAS: Evaluation of 2008-11 HWRF forecasts. Mon. Wea. Rev., 143, 511-523, https://doi.org/10.1175/MWR-D-14-00138.1.

Aksoy, A., S. Lorsolo, T. Vukicevic, K. J. Sellwood, S. D. Aberson, and F. Zhang, 2012: The HWRF Hurricane Ensemble Data Assimilation System (HEDAS) for high-resolution data: The impact of airborne Doppler radar observations in an OSSE. Mon. Wea. Rev., 140,1843-1862, https://doi.org/10.1175/MWR-D-11-00212.1.

- S. D. Aberson, T. Vukicevic, K. J. Sellwood, S. Lorsolo, and X. Zhang, 2013: Assimilation of high-resolution tropical cyclone observations with an ensemble Kalman filter using NOAA/AOML/HRD's HEDAS: Evaluation of the 2008-11 vortex-scale analyses. Mon. Wea. Rev., 141, 1842-1865, https:// doi.org/10.1175/MWR-D-12-00194.1.

Andersson, E., and M. Masutani, 2010: Collaboration on observing system simulation experiments (Joint OSSE). ECMWF Newsletter, No. 123, ECMWF, Reading, United Kingdom, 14-16, http://www.emc.ncep.noaa.gov/research/ JointOSSEs/publications/JOSSE-Publication-files/Andersson_ JOSSE_ECMWF_News_No123.pdf.

Arnold, C. P., and C. H. Dey, 1986: Observing-system simulation experiments: Past, present, and future. Bull. Amer. Meteor. Soc., 67, 687-695, https://doi.org/10.1175/1520-0477(1986) 067<0687:OSSEPP>2.0.CO;2.

Atlas, R., 1997: Atmospheric observations and experiments to assess their usefulness in data assimilation. J. Meteor. Soc. Japan, 75, 111-130, https://doi.org/10.2151/jmsj1965.75.1B_111.

Black, M. L., and H. E. Willoughby, 1992: The concentric eyewall cycle of Hurricane Gilbert. Mon. Wea. Rev., 120, 947-957, https:// doi.org/10.1175/1520-0493(1992)120<0947:TCECOH>2.0.CO;2.

Cangialosi, J., T. Kimberlain, J. Beven, and M. DeMaria, 2015: The validity of Dvorak intensity change constraints for tropical cyclones. Wea. Forecasting, 30, 1010-1015, https://doi.org/ 10.1175/WAF-D-15-0028.1.

Chavas, D. R., and K. A. Emanuel, 2010: A QuikSCAT climatology of tropical cyclone size. Geophys. Res. Lett., 37, L18816, https://doi.org/10.1029/2010GL044558.

Christophersen, H., A. Aksoy, J. Dunion, and K. Sellwood, 2017: The impact of NASA Global Hawk unmanned aircraft dropwindsonde observations on tropical cyclone track, intensity, and structure: Case studies. Mon. Wea. Rev., 145, 1817-1830, https://doi.org/10.1175/MWR-D-16-0332.1.

DeMaria, M., C. R. Sampson, J. A. Knaff, and K. D. Musgrave, 2014: Is tropical cyclone intensity guidance improving? Bull. Amer. Meteor. Soc., 95, 387-398, https://doi.org/10.1175/ BAMS-D-12-00240.1.

Dunion, J. P., 2011: Rewriting the climatology of the tropical North Atlantic and Caribbean Sea atmosphere. J. Climate, 24, 893908, https://doi.org/10.1175/2010JCLI3496.1.

Dvorak, V. F., 1975: Tropical cyclone intensity analysis and forecasting from satellite imagery. Mon. Wea. Rev., 103, 420-430, https://doi.org/ 10.1175/1520-0493(1975)103<0420:TCIAAF > 2.0.CO;2.

_ 1984: Tropical cyclone intensity analysis using satellite data. NOAA Tech. Rep. NESDIS 11, 45 pp., https://severe. worldweather.wmo.int/TCFW/RAI_Training/Dvorak_1984.pdf.

Franklin, J. L., M. L. Black, and K. Valde, 2003: GPS dropwindsonde wind profiles in hurricanes and their operational implications. Wea. Forecasting, 18, 32-44, https://doi.org/10.1175/ 1520-0434(2003)018<0032:GDWPIH >2.0.CO;2.

Gall, R., J. Franklin, F. Marks, E. N. Rappaport, and F. Toepfer, 2013: The Hurricane Forecast Improvement Project. Bull. Amer. Meteor. Soc., 94, 329-343, https://doi.org/10.1175/ BAMS-D-12-00071.1.

Green, B. W., and F. Zhang, 2015: Idealized large-eddy simulations of a tropical cyclone-like boundary layer. J. Atmos. Sci., 72, 1743-1764, https://doi.org/10.1175/JAS-D-14-0244.1.

Hendricks, E. A., B. D. McNoldy, and W. H. Schubert, 2012: Observed inner-core structural variability in Hurricane Dolly (2008). Mon. Wea. Rev., 140, 4066-4077, https://doi.org/10.1175/ MWR-D-12-00018.1.

Hong, S.-Y., Y. Noh, and J. Dudhia, 2006: A new vertical diffusion package with an explicit treatment of entrainment processes. Mon. Wea. Rev., 134, 2318-2341, https://doi.org/ 10.1175/MWR3199.1.

Janjić, Z. I., 1990: The step-mountain coordinate: Physical package. Mon. Wea. Rev., 118, 1429-1443, https://doi.org/10.1175/15200493(1990)118<1429:TSMCPP $>2.0$. CO;2.

, 1994: The step-mountain eta coordinate model: Further developments of the convection, viscous sublayer, and turbulence closure schemes. Mon. Wea. Rev., 122, 927-945, https://doi.org/ 10.1175/1520-0493(1994)122<0927:TSMECM>2.0.CO;2.

Jarvinen, B. R., C. J. Neumann, and M. A. S. Davis, 1984: A tropical cyclone data tape for the North Atlantic basin, 18861983: Contents, limitations, and uses. NOAA Tech. Memo. NWS NHC 22, 21 pp.

Kimball, S. K., and M. S. Mulekar, 2004: A 15-year climatology of North Atlantic tropical cyclones. Part I: Size parameters. J. Climate, 17, 3555-3575, https://doi.org/10.1175/1520-0442(2004) 017<3555:AYCONA $>2.0 . \mathrm{CO} ; 2$.

Klotz, B. W., and E. W. Uhlhorn, 2014: Improved stepped frequency microwave radiometer tropical cyclone surface winds in heavy precipitation. J. Atmos. Oceanic Technol., 31, 23922408, https://doi.org/10.1175/JTECH-D-14-00028.1.

_ , and H. Jiang, 2017: Examination of surface wind asymmetries in tropical cyclones. Part I: General structure and wind shear impacts. Mon. Wea. Rev., 145, 3989-4009, https://doi.org/10.1175/ MWR-D-17-0019.1.

Knaff, J., D. Brown, J. Courtney, G. Gallina, and J. Beven, 2010: An evaluation of Dvorak technique-based tropical cyclone intensity estimates. Wea. Forecasting, 25, 1362-1379, https:// doi.org/10.1175/2010WAF2222375.1.

Kosiba, K. A., and J. Wurman, 2014: Finescale dual-Doppler analysis of hurricane boundary structures in Hurricane Frances (2004) 
at landfall. Mon. Wea. Rev., 142, 1874-1891, https://doi.org/ 10.1175/MWR-D-13-00178.1.

Landsea, C. W., and J. L. Franklin, 2013: Atlantic hurricane database uncertainty and presentation of a new database format. Mon. Wea. Rev., 141, 3576-3592, https://doi.org/10.1175/MWR-D-12-00254.1. , and Coauthors, 2004: A reanalysis of Hurricane Andrew's intensity. Bull. Amer. Meteor. Soc., 85, 1699-1712, https:// doi.org/10.1175/BAMS-85-11-1699.

Lorsolo, S., J. L. Schroeder, P. Dodge, and F. Marks, 2008: An observational study of hurricane boundary layer small-scale coherent structures. Mon. Wea. Rev., 136, 2871-2893, https:// doi.org/10.1175/2008MWR2273.1.

Marks, F. D., 1985: Evolution of the structure of precipitation in Hurricane Allen (1980). Mon. Wea. Rev., 113, 909-930, https:// doi.org/10.1175/1520-0493(1985)113<0909:EOTSOP>2.0.CO;2.

_ , P. G. Black, M. T. Montgomery, and R. W. Burpee, 2008: Structure of the eye and eyewall of Hurricane Hugo (1989). Mon. Wea. Rev., 136, 1237-1259, https://doi.org/10.1175/2007MWR2073.1.

Moon, Y., and D. Nolan, 2015: Spiral rainbands in a numerical simulation of Hurricane Bill (2009). Part I: Structures and comparisons to observations. J. Atmos. Sci., 72, 164-190, https://doi.org/10.1175/JAS-D-14-0058.1.

Nolan, D. S., 2005: Instabilities in hurricane-like boundary layers. Dyn. Atmos. Oceans, 40, 209-236, https://doi.org/10.1016/ j.dynatmoce.2005.03.002.

- 2007: What is the trigger for tropical cyclogenesis? Aust. Meteor. Mag., 56, 241-266, http://www.bom.gov.au/jshess/docs/ 2007/nolan.pdf.

_- 2011: Evaluating environmental favorableness for tropical cyclone development with the method of point-downscaling. J. Adv. Model. Earth Syst., 3, M08001, https://doi.org/10.1029/ 2011MS000063.

— , and M. G. McGauley, 2012: Tropical cyclogenesis in wind shear: Climatological relationships and physical processes. Cyclones: Formation, Triggers and Control, K. Oouchi and H. Fudeyasu, Eds., Nova Science Publishers, 1-34.

— second hurricane nature run using the joint OSSE nature run and the WRF Model. 31st Conf. on Hurricanes and Tropical Meteorology, San Diego, CA, Amer. Meteor. Soc., 91, https:// ams.confex.com/ams/31Hurr/webprogram/Paper244751.html.

— , J. A. Zhang, and D. P. Stern, 2009a: Evaluation of planetary boundary layer parameterizations in tropical cyclones by comparison of in situ observations and high-resolution simulations of Hurricane Isabel (2003). Part I: Initialization, maximum winds, and the outer-core boundary layer. Mon. Wea. Rev., 137, 3651-3674, https://doi.org/10.1175/2009MWR2785.1.

, D. P. Stern, and J. A. Zhang, 2009b: Evaluation of planetary boundary layer parameterizations in tropical cyclones by comparison of in situ observations and high-resolution simulations of Hurricane Isabel (2003). Part II: Innercore boundary layer and eyewall structure. Mon. Wea. Rev., 137, 3675-3698, https://doi.org/10.1175/2009MWR2786.1.

_ R. Atlas, K. T. Bhatia, and L. R. Bucci, 2013: Development and validation of a hurricane nature run using the joint OSSE nature run and the WRF Model. J. Adv. Model. Earth Syst., 5, 382-405, https://doi.org/10.1002/jame.20031.

— J. A. Zhang, and E. W. Uhlhorn, 2014: On the limits of estimating the maximum wind speeds in hurricanes. Mon. Wea. Rev., 142, 2814-2837, https://doi.org/10.1175/MWR-D-13-00337.1.

OFCM, 2012: National Hurricane Operations Plan. U.S. Department of Commerce/National Oceanic and Atmospheric Administration Rep. FCM-P-2012, 168 pp.
Pollard, R. T., P. B. Rhines, and R. Thompson, 1972: The deepening of the wind-mixed layer. Geophys. Fluid Dyn., 4, 381404, https://doi.org/10.1080/03091927208236105.

Powell, M. D., and P. G. Black, 1990: The relationship of hurricane reconnaissance flight-level wind measurements to winds measured by NOAA's oceanic platforms. J. Wind Eng. Ind. Aerodyn., 36, 381-392, https://doi.org/10.1016/0167-6105(90)90322-4. , P. P. Dodge, and M. L. Black, 1991: The landfall of Hurricane Hugo in the Carolinas: Surface wind distribution. Wea. Forecasting, 6, 379-399, https://doi.org/10.1175/1520-0434(1991) $006<0379$ :TLOHHI $>2.0$. CO;2.

— E. W. Uhlhorn, and J. D. Kepert, 2009: Estimating maximum surface winds from hurricane reconnaissance measurements. Wea. Forecasting, 24, 868-883, https://doi.org/10.1175/ 2008WAF2007087.1.

Rogers, R., and Coauthors, 2013: NOAA's Hurricane Intensity Forecasting Experiment: A progress report. Bull. Amer. Meteor. Soc., 94, 859-882, https://doi.org/10.1175/BAMS-D-12-00089.1.

Shapiro, L. J., and H. E. Willoughby, 1982: The response of balanced hurricanes to local sources of heat and momentum. J. Atmos. Sci., 39, 378-394, https://doi.org/10.1175/1520-0469(1982)039<0378: TROBHT > 2.0.CO;2.

Stern, D. P., and D. S. Nolan, 2011: On the vertical decay rate of the maximum tangential winds in tropical cyclones. J. Atmos. Sci. 68, 2073-2094, https://doi.org/10.1175/2011JAS3682.1.

— J. L. Vigh, D. S. Nolan, and F. Zhang, 2015: Revisiting the relationship between eyewall contraction and intensification. J. Atmos. Sci., 72, 1283-1306, https://doi.org/10.1175/JAS-D14-0261.1.

Uhlhorn, E. W., and P. G. Black, 2003: Verification of remotely sensed sea surface winds in hurricanes. J. Atmos. Oceanic Technol., 20, 99-116, https://doi.org/10.1175/1520-0426(2003) $020<0099$ :VORSSS $>2.0$.CO;2.

— tropical cyclones and implications for estimated intensity. Mon. Wea. Rev., 140, 825-840, https://doi.org/10.1175/MWRD-11-00073.1.

_ , P. G. Black, J. L. Franklin, M. Goodberlet, J. Carswell, and A. S. Goldstein, 2007: Hurricane surface wind measurements from an operational stepped frequency microwave radiometer. Mon. Wea. Rev., 135, 3070-3085, https://doi.org/10.1175/ MWR3454.1.

Vickery, P. J., and P. F. Skerlj, 2005: Hurricane gust factors revisited. J. Struct. Eng., 131, 825-832, https://doi.org/10.1061/ (ASCE)0733-9445(2005)131:5(825).

Vigh, J. L., 2010: Formation of the hurricane eye. Ph.D. dissertation, Colorado State University, 538 pp., http://www.ral.ucar.edu/ staff/jvigh/documents/vigh2010_dissertation_corrected_color_ hyperlinks.pdf.

—, J. A. Knaff, and W. H. Schubert, 2012: A climatology of hurricane eye formation. Mon. Wea. Rev., 140, 1405-1426, https://doi.org/10.1175/MWR-D-11-00108.1.

Vukicevic, T., E. Uhlhorn, P. Reasor, and B. Klotz, 2014: A novel multiscale intensity metric for evaluation of tropical cyclone intensity forecasts. J. Atmos. Sci., 71, 1292-1304, https://doi.org/ 10.1175/JAS-D-13-0153.1.

Willoughby, H. E., J. A. Clos, and M. G. Shoreibah, 1982: Concentric eye walls, secondary wind maxima, and the evolution of the hurricane vortex. J. Atmos. Sci., 39, 395-411, https://doi.org/ 10.1175/1520-0469(1982)039<0395:CEWSWM >2.0.CO;2.

Wurman, J., and J. Winslow, 1998: Intense sub-kilometer-scale boundary layer rolls observed in Hurricane Fran. Science, 280, 555-557, https://doi.org/10.1126/science.280.5363.555. 